\title{
Influencia del estiércol composteado y micorriza arbuscular sobre la composición química del suelo y el rendimiento productivo de maíz forrajero (Zea mays L.) \\ Influence of composted manure and arbuscular mycorrhiza on the chemical composition of the soil and yield of maize fodder (Zea mays $\mathrm{L}$.)
}

Manuela Margarita Jiménez Ortiz ${ }^{1}$, Regino Gómez Álvarez ${ }^{1}$, Jorge Oliva Hernández², Lorenzo Granados Zurita ${ }^{2}$, Juan Manuel Pat Fernández ${ }^{3}$ y Emilio Manuel Aranda Ibáñez ${ }^{4}$

Palabras clave: enmiendas orgánicas; biofertilizante; trópico; productividad Keywords: organic amendments; biofertilizer; tropic; productivity

Recibido en: 16-04-2019 / Aceptado en: 19-08-2019

\section{Resumen}

Introducción: El estiércol composteado (E) es una alternativa al reciclaje de nutrientes, y puede mejorar o mantener la fertilidad del suelo sustituyendo a la fertilización convencional. El objetivo del estudio fue evaluar las características y composición química del suelo, porcentaje de infección del hongo micorrízico arbuscular (M) en raíz, características agronómicas de la planta, tasa de crecimiento en el cultivo, acumulación de nutrientes y rendimiento productivo, en respuesta del E y del Glomus intraradices (M) en maíz forrajero.

Método: Se evaluaron seis niveles de fertilización orgánica $\left(15,30\right.$ y $45 \mathrm{t} \mathrm{ha}^{-1}$ de E, 15, 30 y $45 \mathrm{t}$ $\mathrm{ha}^{-1}$ de EM), uno de fertilización química (FQ: 160-60-30 $\mathrm{kg} \mathrm{ha}^{-1}$ de NPK), y un testigo (sin fertilizar: SF), en dos ciclos de cultivos (Ciclo, PV: primavera-verano y OI: otoño-invierno). Se utilizó E y $G$. intraradices. Las variables estudiadas en el suelo fueron: $\mathrm{pH}$, conductividad eléctrica (CE), materia orgánica (MO), N, P, K, Ca, capacidad de intercambio catiónico (CIC), numero de esporas; en la planta: acumulación de nutrientes $(\mathrm{N}, \mathrm{P}, \mathrm{K}, \mathrm{Ca})$, porcentaje de infección en raíz, altura, numero de hojas y materia seca (MS); en el cultivo: tasa de crecimiento del cultivo (TCC), rendimiento de forraje en materia verde ( $\mathrm{MV} \mathrm{tha-1}$ ) y materia seca $\left(\mathrm{MS} \mathrm{tha}^{-1}\right)$.

Resultados: Con excepción del K en suelo y la CIC, el Tratamiento, Ciclo y la interacción de ambos factores afectaron $(P<0.05)$ las variables que definieron la composición química del suelo ( $\mathrm{pH}, \mathrm{CE}, \mathrm{MO}, \mathrm{N}, \mathrm{P}, \mathrm{Ca})$, la acumulación de nutrientes en planta (N, P, K, Ca) y el rendimiento de forraje en MV y MS.

\footnotetext{
${ }^{1}$ El Colegio de la Frontera Sur. E-mail: majimenez@ecosur.edu.mx

${ }^{2}$ Instituto Nacional de Investigaciones Forestales, Agrícolas y Pecuarias, Campo Experimental Huimanguillo

${ }^{3}$ El Colegio de la Frontera Sur, Unidad Campeche

${ }^{4}$ Colegio de Postgraduados, Campus Tabasco

(C) Universidad De La Salle Bajío (México)
} 
En OI, FQ presentó el menor contenido de MO; en PV, un incremento en el E (de 15E a 45E) permitió aumentar la MO $(P>0.05)$. El $\mathrm{N}$ en suelo fue mayor en OI con relación a PV en todos los tratamientos $(P>0.05)$. En OI, 30EM presentó la mayor acumulación de nutrientes en planta $(\mathrm{N}, \mathrm{P}, \mathrm{K}$ y $\mathrm{Ca})$ con respecto al resto de los tratamientos $(P>0.05)$. En ambos ciclos, PV y OI, 30EM tuvo el mayor rendimiento de forraje en MS $(P>0.05)$.

Discusión o Conclusión: La composición química del suelo, planta y el rendimiento de maíz forrajero fueron afectados por el uso de E solo o en combinación con M durante los ciclos PV y OI. El uso de 30EM representa una mejor opción de fertilización con relación SF y FQ debido a que la planta tuvo mayor producción de forraje en MS durante la PV y OI. El uso de $G$. intraradices en combinación con E contribuye a optimizar la utilización de nutrientes del suelo por la planta. Los resultados obtenidos son importantes para incrementar la calidad y cantidad de maíz forrajero.

\section{Abstract}

Introduction: Composted manure $(\mathrm{CM})$ is an alternative to nutrient recycling, and can improve or maintain soil fertility by replacing conventional fertilization. The objective of the study was to evaluate the characteristics and chemical composition of the soil, percentage of infection of the arbuscular mycorrhizal fungus (AMF) in the root, agronomic characteristics of the plant, growth rate in the crop, nutrient accumulation and productive yield, in response to the E and Glomus intraradices $(\mathrm{CM})$ in forage corn.

Method: Six levels of organic fertilization were evaluated (15, 30 and $45 \mathrm{t} \mathrm{ha}^{-1}$ of E, 15, 30 and $45 \mathrm{t} \mathrm{ha}^{-1}$ of EM), one of chemical fertilization (QF: 160-60-30 kg) ha-1 of NPK), and a control (without fertilizing: WF), in two crop cycles (Cycle, SS: spring-summer and AW: autumnwinter). E and G. intraradices were used. The variables studied in the soil were: $\mathrm{pH}$, electrical conductivity (EC), organic matter (OM), N, P, K, Ca, cation exchange capacity (CEC), number of spores; in the plant: accumulation of nutrients $(\mathrm{N}, \mathrm{P}, \mathrm{K}, \mathrm{Ca})$, percentage of infection in root, height, number of leaves and dry matter (DM); in the crop: growth rate of the crop (GRC), yield of forage in green matter $\left(\mathrm{GM} \mathrm{tha} \mathrm{th}^{-1}\right)$ and dry matter $\left(\mathrm{DM} \mathrm{tha} \mathrm{h}^{-1}\right)$.

Results: With the exception of the $\mathrm{K}$ in soil and the CEC, the Treatment, Cycle and the interaction of both factors affected $(P<0.05)$ the variables that defined the chemical composition of the soil ( $\mathrm{pH}, \mathrm{EC}, \mathrm{OM}, \mathrm{N}, \mathrm{P}, \mathrm{Ca})$, the accumulation of plant nutrients $(\mathrm{N}, \mathrm{P}, \mathrm{K}, \mathrm{Ca})$ and forage 
yield in GM and DM. In OI, QF presented the lowest OM content; in SS, an increase in E (from $15 \mathrm{E}$ to $45 \mathrm{E})$ allowed to increase the $\mathrm{OM}(P<0.05)$. The $\mathrm{N}$ in soil was higher in $\mathrm{AW}$ in relation to SS in all treatments $(P<0.05)$. In AW, 30EM presented the highest accumulation of plant nutrients $(\mathrm{N}, \mathrm{P}, \mathrm{K}$ and $\mathrm{Ca})$ with respect to the rest of the treatments $(P<0.05)$. In both cycles, SS and AW, 30EM had the highest forage yield in DM $(P<0.05)$.

Discussion or Conclusion: The chemical composition of the soil, plant and forage maize yield were affected by the use of $\mathrm{E}$ alone or in combination with $\mathrm{M}$ during the SS and AW cycles. The use of 30EM represents a better fertilization option with relation WF and QF because the plant had more forage production in MS during the SS and AW. The use of G. intraradices in combination with $\mathrm{E}$ contributes to optimize the use of soil nutrients by the plant. The results obtained are important to increase the quality and quantity of feed corn.

\section{Introducción}

El cultivo de maíz se desarrolla en México mayormente bajo un agroecosistema convencional con paquetes tecnológicos que están basados en el uso de insumos externos (Damián et al., 2010, 68). En la zona tropical húmeda de México, el cultivo del maíz para producción de forraje y su subsecuente ensilaje se ha incrementado en los últimos cinco años en un 20\% (SIAP, 2018). La conservación de follaje de la planta a través del ensilaje y su posterior uso en la alimentación del ganado bovino en regiones no tropicales es una práctica común (Garcés et al., 2004, 67). Sin embargo, en la región tropical húmeda de México, el ensilaje de maíz es relativamente reciente, por lo que se debe generar tecnología orientada a optimizar su rendimiento productivo y calidad nutricional (Barrón et al., 2014, 312). La elaboración de silo de maíz se considera como un reservorio en la alimentación de rumiantes en épocas de escases de forrajes como la sequía y nortes, o como complementación durante todo el año. Debido a esto, el cultivo de maíz forrajero en el trópico húmedo se realiza tanto en el ciclo Primavera - Verano, como en Otoño - Invierno para cubrir las necesidades de alimentación del ganado (Barrón et al., 2014, 311). En el proceso de cosecha del maíz forrajero se remueve la planta completa y por consiguiente no hay residuos de la cosecha que pudieran reincorporarse al suelo, lo que reduce la disponibilidad de MO como sustrato para el desarrollo y funcionamiento de los microorganismos del suelo (Gliessman et al., 2007, 16). Los nutrimentos que requiere la planta de maíz bajo un manejo convencional del 
cultivo son aportados generalmente por fertilizantes inorgánicos. Con ello se espera obtener un alto rendimiento de biomasa por ciclo de cultivo (Díaz et al., 2014, 35). Sin embargo, es importante considerar que el uso excesivo de insumos agrícolas genera daños ecológicos por reducir la eficiencia del uso de nutrientes aplicados (Shanahan et al., 2008, 52-53), así como, afectar la fertilidad del suelo por extracción de nutrientes, compactar el suelo y disminuir el contenido de materia orgánica, la cual es más rápida que su acumulación (Diacono y Montemurro, 2010, 403).

En la productividad del suelo, la MO es un reservorio importante de carbono (C) y fuente de nutrientes que requieren las plantas para su crecimiento como el Nitrógeno (N), con aproximadamente más del $90 \%$ en sus formas orgánicas (Sparling et al., 2006, 548). Sus componentes se encuentran en un estado dinámico debido a que por las diversas transformaciones contantemente pasan de un estado a otro. La descomposición de la MO está relacionada con la mineralización, la dinámica de la materia orgánica disuelta, la humificación y la estabilización de la MO. Esta dinámica de transformaciones se puede ver influenciada por factores climáticos, físicos, químicos y por la calidad del origen de la MO (Zech et al., 1997, 118). La liberación de nutrientes aportados al suelo se da a través de la descomposición y mineralización de la MO. La mineralización es la transformación de los elementos orgánicos a compuestos inorgánicos, con la intervención de la actividad microbiana contenida en el suelo. Dichas transformaciones se dan a diferentes velocidades dependiendo de las fracciones de la $\mathrm{MO}$, rápida en los componentes lábiles en una primera fase de descomposición y lenta en una segunda fase debido a moléculas refractarias (resistentes). La mineralización de la MO está influenciada por la temperatura y la humedad, entre otros factores como el pH, la calidad de los componentes primarios, etc., por lo que en regiones cálidas la acumulación de la MO se ve disminuida por una rápida mineralización y disponibilidad de nutrientes. Lo anterior es debido a que los microbios, quienes son los encargados de los procesos de degradación de la MO, son los directamente afectados por dichos factores (Zech et al., 1997, 133; Gallardo, 2001, 146).

La preparación del suelo realizado para los cultivos disminuye las entradas de C y el bajo contenido de MO (por aumento de la tensión de Oxígeno que acelera la mineralización), no obstante, la aplicación de prácticas agroecológicas, tales como, el uso de abonos orgánicos (estiércol composteado), pueden evitar estos perjuicios (Gallardo, 2001, 146). Los abonos orgánicos son una alternativa para incorporar nutrientes al suelo y planta. Si bien una de las 
desventajas de estos, es la lenta disponibilidad de nutrientes a las plantas y la acumulación de sales (Castro et al., 2009, 32; Quiroga et al., 2011, 202). Sin embargo, los beneficios por adicionar materia orgánica al suelo pueden ser mayores, destacando el aumento del contenido de carbono, una mayor capacidad de intercambio catiónico (la cual es importante para la disponibilidad de nutrientes de las plantas), una mejor relación $\mathrm{C} / \mathrm{N}$ y mayor disponibilidad de potasio y fósforo (Diacono y Montemurro, 2010, 409, 410). El estiércol composteado contiene residuos fácilmente degradables, con efecto intenso y transitorio, y residuos más resistentes como la lignina y la celulosa, con un efecto menor pero duradero (Diacono y Montemurro, 2010, 411). Respecto al rendimiento en el cultivo, éste se ha relacionado con el contenido de carbono en el suelo, pero depende de factores como el contenido inicial de este elemento, manejo del suelo, uso de fertilizantes inorgánicos y orgánicos, entre otros (Martínez et al., 2008, 86). El contenido de MO en el suelo, es un indicador que se relaciona con su calidad y productividad. El uso de abonos orgánicos de forma continua puede aumentar la MO significativamente en suelos con niveles bajos (Ramos y Terry, 2014, 53).

Otra práctica agroecológica que puede beneficiar la producción de un sistema es el uso del hongo micorrízico arbuscular (M) como el Glomus intraradices, que propicia una relación simbiótica con la mayoría de las plantas terrestres. Esta asociación entre hongo-planta, genera una extensión radical que propicia mayor absorción de nutrientes para la planta como el P, fijación del $\mathrm{N}_{2}$, mejora la calidad del suelo y aumenta la diversidad y productividad de las plantas en diferentes ecosistemas. Se ha argumentado que las prácticas de la agricultura convencional han generado disminución en las poblaciones de M, influyendo también en la calidad del suelo y su productividad (Barrer, 2009, 124). En trabajos realizados con la aplicación de fertilización orgánica en el cultivo de maíz forrajero, se obtuvo una producción de forraje similar a la fertilización convencional y superior al testigo (Trejo et al., 2013, 735; López et al., 2010, 53) o superiores respecto a la fertilización convencional (Salazar et al., 2009, 377). Si bien no siempre se obtienen rendimientos superiores con la fertilización orgánica, si pueden ser similares al uso de fertilizantes convencionales (Trejo et al., 2013, 735). Estudios realizados con el M Glomus intraradices mostraron incrementos en los rendimientos en grano de maíz (17.6\%) al combinarse con humus de lombriz (Pérez, 2012, 56) y del 29.9\% respecto al testigo cuando se combina con Azospirillum (Uribe et al., 2007, 13). 
En la zona donde se realizó el presente estudio, (Vega del Río Mezcalapa en Tabasco, México) se desarrolla el sistema de producción (SP) bovinos de doble propósito (carne y leche) tradicional. Estas unidades de producción cuentan con alrededor de $20 \pm 9.01$ vacas en producción en promedio. La alimentación del ganado es a base de forrajes con pastoreo extensivo y pocos complementan con ensilaje de maíz. Su sistema de ordeña es manual y su índice tecnológico es de $5 \pm 3.89$, el cual hace referencia a que presentan una baja adopción de tecnologías en sus SP (Granados-Rivera et al., 2018, 50-51). Una de las desventajas de este sistema es la generación y acumulación del estiércol en los corrales de ordeña (52 t estiércol seco año $^{-1}$ aproximadamente). Esta situación representa un problema de manejo para el productor quien frecuentemente desconoce algunas alternativas para el manejo, procesamiento y reciclado del estiércol de los animales (Sánchez et al., 2011, 377). Ante este escenario, es importante realizar prácticas de manejo que puedan mostrar al productor el beneficio del reciclaje del estiércol y su uso como fertilizante orgánico en la producción de maíz forrajero (el cual emplean para ensilar).

La implementación de prácticas agroecológicas como la fertilización orgánica (estiércol composteado) y el uso de microorganismos benéficos como el M, pueden beneficiar los sistemas de producción agropecuarios. Esto debido a que el uso del estiércol composteado es una alternativa al reciclaje de nutrientes, y puede mejorar o mantener la fertilidad del suelo al emplearlo como sustitución a la fertilización convencional. En base a lo anterior, el objetivo de la presente investigación fue evaluar la composición química del suelo, número de esporas de $\mathrm{M}$ en el suelo, variables agronómicas, porcentaje de infección del $\mathrm{M}$ en raíz, tasa de crecimiento del cultivo (TCC), nutrientes acumulados y productividad del cultivo, empleando estiércol composteado (E) y $\mathrm{M}$ en dos ciclos de cultivo de maíz forrajero bajo condiciones de trópico húmedo de Tabasco.

\section{Método}

\section{Localización}

El estudio se realizó en Huimanguillo, Tabasco, México (15Q 0456225; 1948685 UTM). El clima de la región es cálido húmedo con lluvias en verano Am(w)',(i) g (García, 2004, 39), con influencia de nortes en invierno y ligera sequía en los meses de agosto y septiembre (llamada canícula). La época de lluvias se presenta de junio a septiembre, período en el cual se concentra 
el $71.5 \%$ de la precipitación pluvial anual. Un periodo seco entre marzo y abril con $39 \%$ de evaporación. La temporada regular de nortes se extiende de octubre a marzo (West et al., 1985, 34). La temperatura ambiente media anual es de $26.7{ }^{\circ} \mathrm{C}$ con máxima de $39.7{ }^{\circ} \mathrm{C}$ y mínima de 13.2 ${ }^{\circ} \mathrm{C}$. El suelo corresponde a la denominación Fluvisol éutrico (Palma et al., 2007, 26), de textura arcillo-arenoso, localmente conocido como suelo de vega de río, con pH de 4.6 a 5.4.

El estudio se realizó durante dos ciclos de cultivo Primavera-Verano (PV) 2017 (junioseptiembre) y Otoño-Invierno (OI) 2017/2018 (diciembre, enero, febrero y marzo) bajo condiciones exclusivamente de temporal. Los datos climatológicos de precipitación pluvial acumulada $\left(\mathrm{mm} \mathrm{día} \mathrm{a}^{-1}\right)$ y temperatura ambiente $\left({ }^{\circ} \mathrm{C} \mathrm{día}^{-1}\right)$ que prevalecieron durante el periodo de estudio, se obtuvieron de la estación meteorológica del Campo Experimental Huimanguillo del Instituto Nacional de Investigaciones Forestales, Agrícolas y Pecuarias (INIFAP) (Fig. 1).

\section{Diseño experimental y Manejo del cultivo}

Se utilizaron dos diseños experimentales. El primer diseño fue factorial con dos factores. El primer factor fue Tratamiento con ocho niveles; seis de fertilización orgánica $\left(15,30\right.$ y $45 \mathrm{t} \mathrm{ha}^{-1}$ de E, 15, 30 y $45 \mathrm{t} \mathrm{ha}^{-1}$ de $\mathrm{E}+\mathrm{M}$ ), uno de fertilización inorgánica (FQ: 160-60-30 $\mathrm{kg} \mathrm{ha}^{-1} \mathrm{de}$ NPK), y un testigo (SF). El segundo factor fue el ciclo de cultivo (PV y OI). El segundo diseño experimental fue factorial con tres factores, en donde los factores: Tratamiento (ocho tratamientos de fertilización) y Ciclo (PV y OI) fueron similares a lo indicado en el primer diseño experimental. El tercer factor fue los días después de la siembra (DDS, 20, 40, 60 y 80 días). Se utilizaron seis repeticiones por tratamiento, cada repetición correspondió a una parcela experimental. Las parcelas experimentales fueron de $20 \mathrm{~m}^{2}$, las cuales contaron con seis surcos, desechando un surco de cada lateral, considerando los cuatro surcos centrales como área útil de cada parcela.

El cultivo de maíz se realizó en un área de 1,140.8 m². Se utilizó el híbrido PAS-525 una distancia de $80 \mathrm{~cm}$ entre surcos y $15 \mathrm{~cm}$ entre plantas, con una densidad de siembra de 82, 500 plantas $\mathrm{ha}^{-1}$. Se uso el paquete tecnológico del INIFAP (preparación del terreno, siembra, control de plagas, etc.) para la producción de maíz para la zona (Tinoco et al., 2002, 13-61). 

productivo de maíz forrajero (Zea mays L.)

Cuadro 1. Descripción de los tratamientos experimentales aplicados en el cultivo de maíz.

\begin{tabular}{|c|c|}
\hline Tratamiento & Descripción \\
\hline SF & Testigo (sin fertilización) \\
\hline $15 \mathrm{E}$ & $15 \mathrm{t} \mathrm{ha}^{-1}$ de estiércol composteado (E) \\
\hline $30 \mathrm{E}$ & $30 \mathrm{t} \mathrm{ha}^{-1}$ de $\mathrm{E}$ \\
\hline $45 \mathrm{E}$ & $45 \mathrm{tha}^{-1}$ de $\mathrm{E}$ \\
\hline $15 \mathrm{EM}$ & $15 \mathrm{t} \mathrm{ha}^{-1}$ de $\mathrm{E}+$ hongo micorrízico arbuscular $G$. intraradices $(\mathrm{M})$ \\
\hline 30EM & $30 \mathrm{t} \mathrm{ha}^{-1}$ de estiércol composteado $+\mathrm{M}$ \\
\hline 45EM & $45 \mathrm{t} \mathrm{ha}^{-1}$ de estiércol composteado $+\mathrm{M}$ \\
\hline FQ & Fertilización química: $160-60-30 \mathrm{~kg}$ de NPK ha-1 \\
\hline
\end{tabular}

Se empleó una dosis de fertilización química (160-60-30 kg de NPK ha ${ }^{-1}$ ) recomendada para el cultivo del maíz en la zona de vega de río de Huimanguillo, Tabasco (Barrón et al., 2014, 312). El fertilizante inorgánico estuvo compuesto por urea $(46 \% \mathrm{~N})$, superfosfato triple de calcio (46\% P) y cloruro de potasio $(60 \% \mathrm{~K})$. La mitad de la dosis de $\mathrm{N}$ y la dosis total del $\mathrm{P}$ y el $\mathrm{K}$, se aplicaron el día de la siembra, de forma manual a chorrillo a lado de cada surco y cubriéndolo con tierra. Posteriormente a los 30 DDS se aplicó el resto de la dosis de $\mathrm{N}$ de igual manera. Se evaluaron tres dosis de fertilización orgánica con estiércol de bovino composteado (E). La dosis del fertilizante orgánico utilizada se determinó por regla de tres, calculando el aporte del 100\% del nivel de $\mathrm{N}$ recomendado para el cultivo (30E). Las dosis de $15 \mathrm{E}$ y $45 \mathrm{E}$ fueron considerados $50 \%$ abajo y $50 \%$ arriba del $100 \%$ de la dosis recomendada para el cultivo (30E). Esta fertilización se realizo un día antes de la siembra de forma manual al voleo y posteriormente se dio un pase de rastra para incorporarlo al suelo. Para el cálculo de la dosis 30E se consideró un aporte de $\mathrm{N}$ del estiércol de 1.5\% y una tasa de liberación del 35\% (Trinidad, 2000, 5-6). La fórmula utilizada para calcular la dosis de estiércol fue la siguiente:

$$
D_{e s t}=\left(D N * t_{e s t}\right) / N_{l i b}
$$

Donde:

$\mathrm{D}_{\text {est }}=$ Dosis de estiércol $\left(\mathrm{t} \mathrm{ha}^{-1}\right)$

$\mathrm{DN}=$ Dosis de $\mathrm{N}$ requerida

$\mathrm{t}_{\text {est }}=1$ tonelada de estiércol

$\mathrm{N}_{\text {lib }}=\mathrm{N}$ liberado en 1 tonelada de estiércol

Considerando que el $\mathrm{N}$ liberado en 1 tonelada de estiércol (con $1.5 \%$ de $\mathrm{N}$ ) es de $5.25 \mathrm{~kg}$ de $\mathrm{N}$, la dosis estimada con la formula anterior fue de $30.4 \mathrm{t} \mathrm{ha}^{-1}$ ajustándose a $30 \mathrm{t} \mathrm{ha}^{-1}$. El estiércol 
utilizado fue recolectado de una unidad de producción de bovinos de doble propósito localizado en la misma zona, el material se encontraba acumulado por varios meses por lo que ya presentaba un precompostaje. Después de extraerlo del sitio donde se encontraba se trabajó en pila de compostaje. La composta fue trabajada cada tres días durante un mes y posteriormente se dejó en reposo durante 30 días.

Los análisis de la composta se realizaron bajo los procedimientos establecidos en la NOM-021-RECNAT-2000 (SEMARNAT, 2002). La composta de estiércol elaborada tuvo la siguiente composición química: $\mathrm{pH}$ : 6.21, conductividad eléctrica (CE): $1.32 \mathrm{dS} \mathrm{m}^{-1}$, materia orgánica (MO): $83.6 \%$, nitrógeno total (Nt): $0.80 \%$, P: $0.36 \%, \mathrm{~K}: 0.76 \%$, Ca: $0.73 \%$ y capacidad de intercambio catiónico (CIC): $24.1 \%$. Respecto al contenido de nutrientes en el suelo inicial, este presentó un pH de 5.77, Nt: 0.22 \%, MO: $2.5 \%$ y P: $29.78 \mathrm{mg} \mathrm{kg}^{-1}$; contenido medio en K $\left(0.36 \mathrm{cmol} \mathrm{kg}^{-1}\right)$ y $\mathrm{Ca}\left(9.67 \mathrm{cmol} \mathrm{kg}^{-1}\right)$ y CIC con $15.3 \mathrm{cmol} \mathrm{kg}$.

Para los tratamientos con micorriza se utilizó el biofertilizante del INIFAP, el cual contiene hongos formadores de micorrizas, con un mínimo de 40 propágulos de $G$. intraradices por gramo de sustrato a base de suelo esterilizado y raíces molidas de gramíneas inoculadas con micorriza. Se utilizó la dosis recomendada a razón de $0.5 \mathrm{~kg}$ de biofertilizante ha ${ }^{-1}$. Las semillas de maíz fueron inoculadas con el biofertilizante un día antes de la siembra. El procedimiento se realizó bajo sombra y consistió en mezclar un adherente en gel con agua, ésta mezcla fue agregada a las semillas para humedecerlas. Inmediatamente se les añadió el biofertilizante a las semillas y se mezcló bien a modo de que quedaran impregnadas de éste. Las semillas fueron puestas a secar sobre una lona para su posterior utilización.

\section{Variables evaluadas}

En el suelo se evaluó el pH, CE, MO, número de esporas, Nt, P, K, Ca y CIC. En planta se evaluó concentración de $\mathrm{Nt}, \mathrm{P}, \mathrm{K}$ y Ca, altura, número de hojas, materia seca (MS) y porcentaje de infección de $\mathrm{M}$ en raíz. En el cultivo se evaluó la tasa de crecimiento del cultivo (TCC), acumulación de Nt, P, K y Ca en la materia seca, rendimiento total en materia verde (MV) y MS. $\mathrm{El}$ análisis de $\mathrm{MO}$ del E se realizó por el método de calcinación (LOI) a $500{ }^{\circ} \mathrm{C}$ por 12 horas. El conteo de esporas de $\mathrm{M}$ se realizó por el método de tamizado húmedo y decantación, posteriormente centrifugado en gradiente de sacarosa. Los análisis químicos de suelo y planta se 
realizaron bajo los procedimientos establecidos en la NOM-021-RECNAT-2000 (SEMARNAT, 2002).

Las evaluaciones finales en la planta se realizaron a la etapa fisiológica de 1/3 de línea de leche (80 días de edad de la planta aproximadamente), edad recomendada para su cosecha y posterior ensilaje (Wiersma et al., 1993, 24; Pigurina y Pérez, 1994, 7-8). Se seleccionaron dos plantas por parcela (12 por tratamiento) de los surcos centrales eliminando el efecto de orilla, a las cuales se les midió la altura con un estadal y el número de hojas, posteriormente fueron sacadas de raíz con todo y suelo a una profundidad de $20 \mathrm{~cm}$ con la ayuda de una pala recta, seguidamente se les cortó la parte aérea la cual fue pesada en verde para calcular el rendimiento total en MV, posteriormente fueron secadas (en estufa de aire forzado por $72 \mathrm{~h}$ a $70{ }^{\circ} \mathrm{C}$ ) para determinar la MS y el rendimiento en MS. Finalmente fueron procesadas en un molino ThomasWiley con malla de $0.5 \mathrm{~mm}$ para la determinación de concentración de nutrientes. Las raíces fueron identificadas, lavadas y secadas para determinar el porcentaje de colonización por micorrizas mediante la técnica de tinción con azul tripano en lactoglicerol (Phillips y Hayman, 1970). Se empleó la planta completa debido a que es utilizada en su totalidad para la práctica del ensilaje. El suelo obtenido de las raíces de las dos plantas por parcela fue secado a la sombra y molido obteniendo una muestra compuesta de $350 \mathrm{~g}$ para analizar su composición química y el conteo de esporas de M. La acumulación de nutrientes en la MS se calculó a partir del rendimiento total en MS obtenido por unidad de superficie multiplicado por la concentración de nutrimentos determinado en la planta total. Las muestras de suelo y plantas se procesaron y analizaron en el laboratorio de Biogeoquímica de El Colegio de la Frontera Sur Unidad Villahermosa. Para la variable TCC se realizaron evaluaciones destructivas de plantas a los 20 , 40, 60 y 80 DDS. Se colectaron dos plantas por parcela de los surcos centrales, fueron cortadas al ras del suelo, pesadas en verde y en seco (en estufa de aire forzado por $72 \mathrm{~h}$ a $70{ }^{\circ} \mathrm{C}$ ). Para estimar la TCC se utilizó la formula (Aguilar et al., 2015, 53):

$$
T C C=\left[\left(P S_{2}-P S_{1}\right) / A\left(T_{2}-T_{1}\right)\right]
$$

Donde:

$\mathrm{TCC}=$ Tasa de Crecimiento del Cultivo

$\mathrm{PS}_{2}=$ Peso Seco Final de la Planta

$\mathrm{PS}_{1}=$ Peso Seco Inicial de la Planta 
T2= Tiempo Final

$\mathrm{T} 1=$ Tiempo Inicial

$\mathrm{A}=$ Área ocupada por la planta

\section{Análisis estadísticos}

Los análisis de datos se efectuaron con apoyo del paquete estadístico SAS (SAS, 2013). El modelo que describió las varianzas de las variables estudiadas en el suelo ( $\mathrm{pH}, \mathrm{CE}, \mathrm{MO}, \mathrm{P}, \mathrm{K}$, $\mathrm{Ca}$, CIC, contenido de esporas de $\mathrm{M}$; en la planta: porcentaje de infección de $\mathrm{M}$ en raíz, acumulación de nutrientes $(\mathrm{N}, \mathrm{P}, \mathrm{K}, \mathrm{Ca})$, altura, número de hojas, MS y rendimiento total de forraje en MV t ha ${ }^{-1}$ y MS t ha ${ }^{-1}$. Se consideró las variables independientes tratamiento, ciclo y su interacción. El modelo que describió la varianza del crecimiento del cultivo incluyó como variables independientes tratamiento, DDS, ciclo y todas las interacciones de primer y segundo orden. Los análisis de datos se efectuaron con el procedimiento GLM. Las medias se compararon con la prueba de " $\mathrm{t}$ " con las medias de cuadrados mínimos usando la opción PDIFF de SAS.

\section{$\underline{\text { Resultados }}$}

Durante el ciclo productivo PV la precipitación pluvial (PP) acumulada fue de $910.9 \mathrm{~mm}$, y un promedio de temperatura registrado de $29.1{ }^{\circ} \mathrm{C}$. Para el ciclo OI, la precipitación pluvial acumulada disminuyó en un $51.6 \%$ con relación a la registrada en PV, y el promedio de temperatura fue de $25^{\circ} \mathrm{C}$ (Fig. 1).
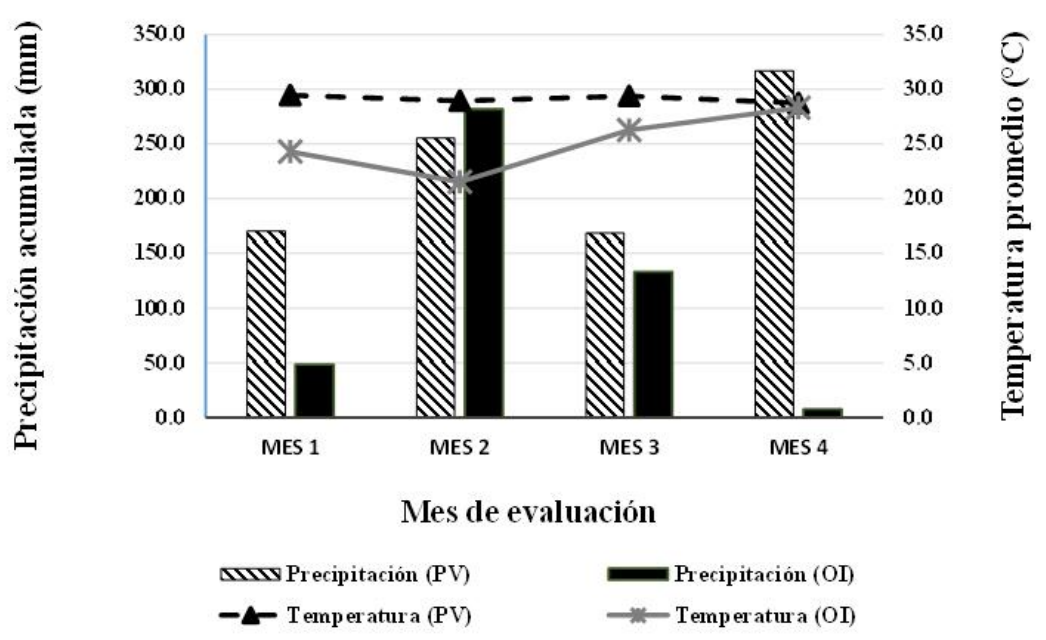

Fig. 1. Precipitación pluvial acumulada y promedio (mensual) de temperatura ambiente en el periodo de estudio de dos ciclos de cultivo del maíz: Primavera - Verano 2017 (junio, julio, agosto y septiembre) y Otoño - Invierno 2017/2018 (diciembre, enero, febrero y marzo). 


\section{Características y composición química del suelo}

Con excepción del K en suelo y la CIC, el Tratamiento, Ciclo y la interacción de ambos factores afectaron $(P<0.05)$ las variables que definieron las características y composición química del suelo ( $\mathrm{pH}, \mathrm{CE}, \mathrm{MO}, \mathrm{N}, \mathrm{P}, \mathrm{Ca}$ y contenido de esporas de M). Por tal motivo se pondrá énfasis en los resultados de la interacción tratamiento x ciclo (Fig. 2).
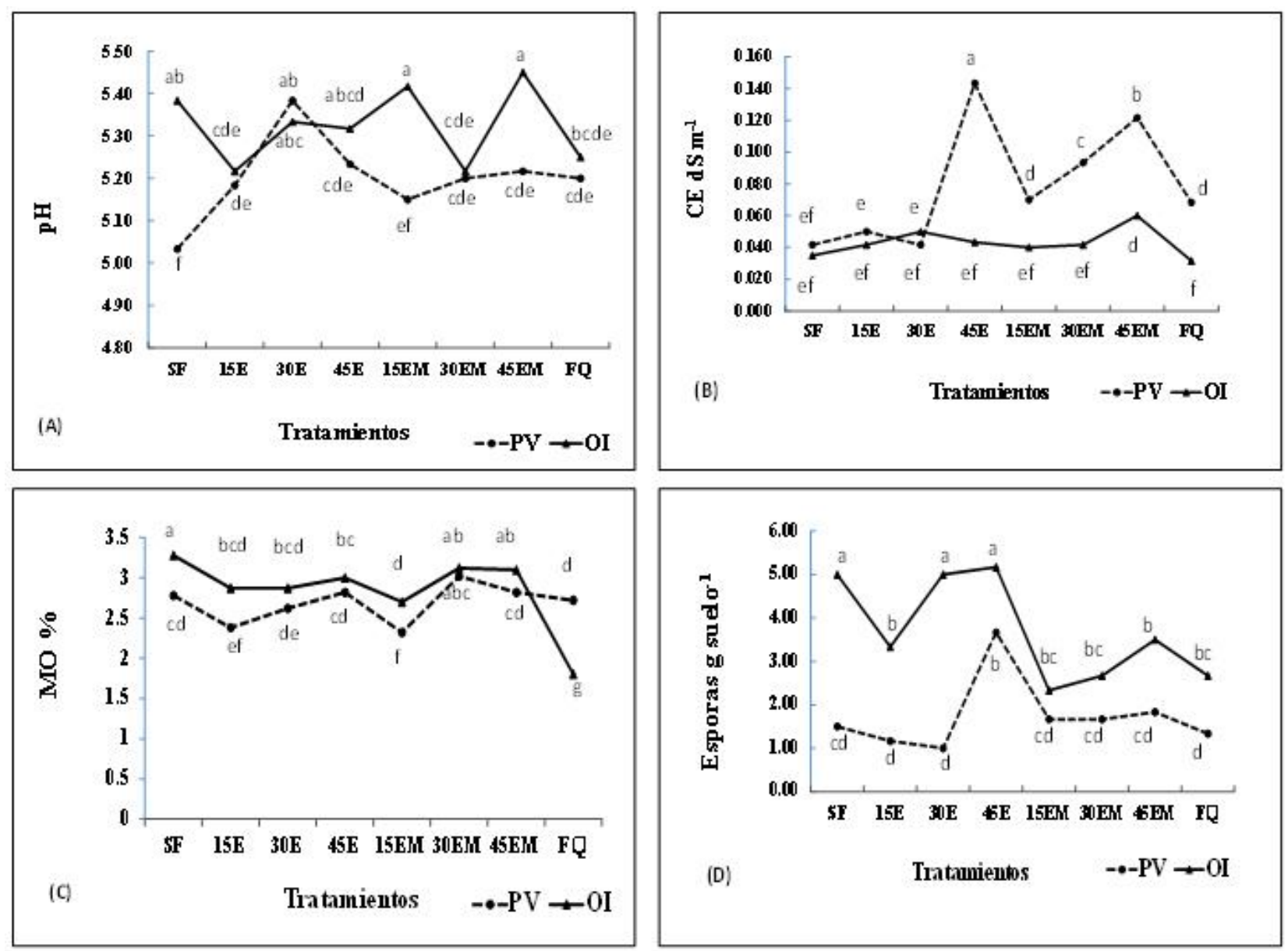

Fig. 2. Efecto de la fertilización con estiércol composteado (E) y del hongo micorrízico arbuscular (M) Glomus intraradices en maíz forrajero sobre el $\mathrm{pH}, \mathrm{CE}$, MO y número de esporas en el suelo en dos ciclos de cultivo (PV y OI).

Literales distintas entre ciclo y entre tratamientos indican diferencia $(P<0.05)$. $\mathbf{C E}=$ Conductividad Eléctrica; $\mathbf{M O}=$ Materia Orgánica; $\mathbf{S F}=$ Sin Fertilizar; 15E=15 t de $E$ ha $^{-1} ; \mathbf{3 0 E}=30$ t de $E$ ha $^{-1} ; \mathbf{4 5 E}=45$ t de $E$ ha $^{-1} ; \mathbf{E M}=E$ Más $\mathrm{M} ; \mathbf{F Q = F e r t i l i z a c i o ́ n ~}$ Química (160-60-30 $\mathrm{kg} \mathrm{ha}^{-1}$ de NPK); PV=Primavera-Verano; OI=Otoño-Invierno.

$\mathrm{El} \mathrm{pH}$ fue menor en SF durante PV con relación al resto de los tratamientos $(P<0.05)$. En PV el $\mathrm{pH}$ del suelo en $15 \mathrm{E}, 30 \mathrm{E}$ y $45 \mathrm{E}$ fue similar $(P>0.05)$ con respecto a estos mismos tratamientos en OI. Sin embargo, en 15EM y 45EM el pH se incrementó en OI con respecto a PV $(P<0.05)$ 
(Fig. 2A). Durante la PV, los tratamientos 45E y 45EM incrementaron la CE con respecto al resto de los tratamientos $(P<0.05)$. Durante el OI, la CE en los tratamientos 45E, 15EM, 30EM, 45EM y FQ fue mayor $(P<0.05)$ con respecto a estos mismos tratamientos en PV (Fig. 2B). En OI y FQ, se detectó el menor contenido de MO $(P<0.05)$; en PV, la MO fue menor en los tratamientos 15E, 15EM y 45EM $(P<0.05)$ con relación a estos mismos tratamientos en OI (Fig. 2C). En los tratamientos sin $\mathrm{M}$, el número de esporas se incrementó en OI con relación a $\mathrm{PV}(P<0.05)$. Con excepción de 45EM, el número de esporas en 15EM y 30EM fue similar durante PV y OI $(P>0.05)$ (Fig. 2D).
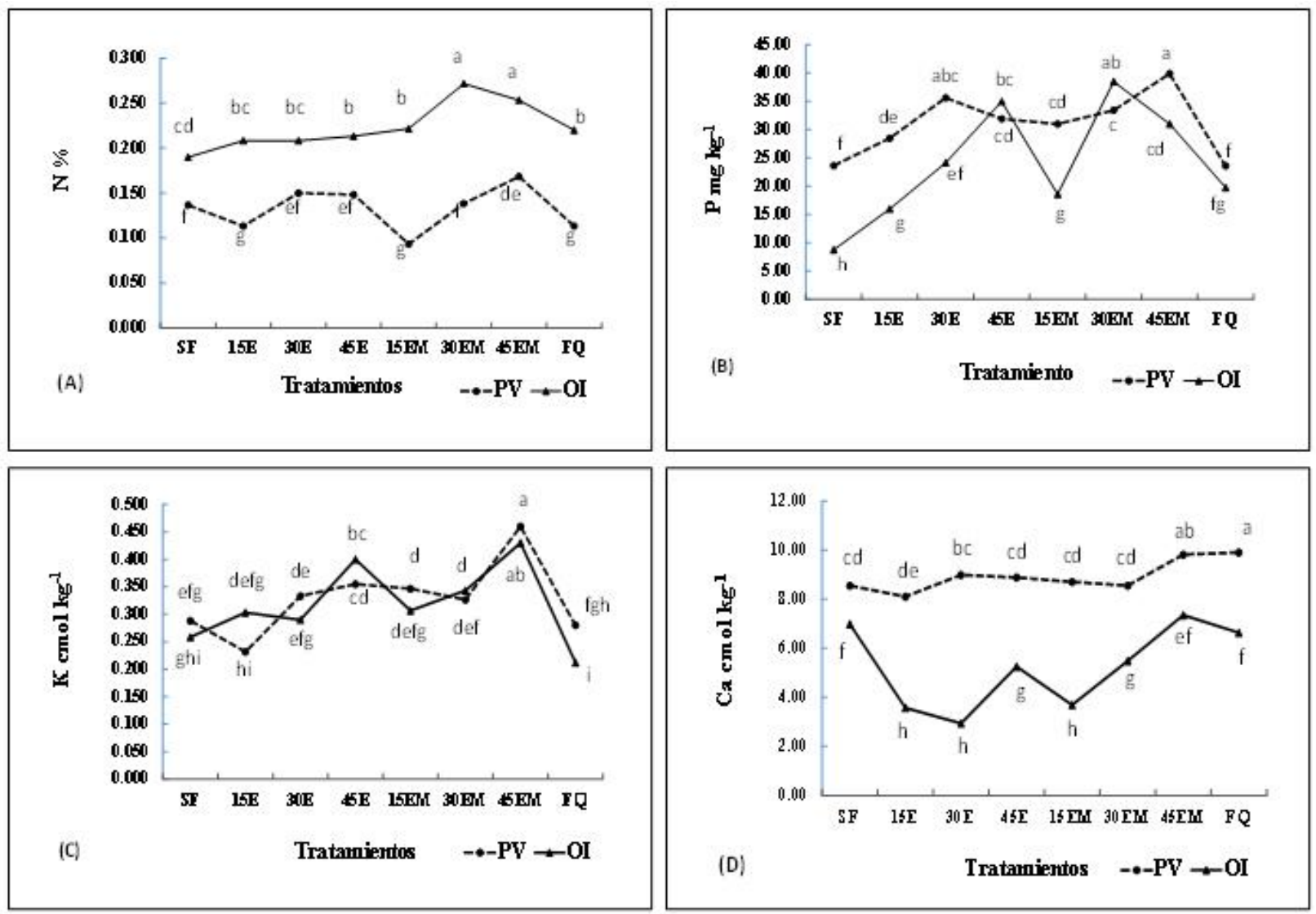

Fig. 3. Efecto de la fertilización con estiércol composteado (E) y micorriza (M) Glomus intraradices en maíz forrajero sobre los niveles de N, P, K y Ca en el suelo en dos ciclos de cultivo (PV y OI).

Literales distintas entre ciclo y entre tratamientos indican diferencia $(P<0.05)$. $\mathbf{S F}=$ Sin Fertilizar; $15 \mathrm{E}=15 \mathrm{t}$ de $\mathrm{E} \mathrm{ha}{ }^{-1} ; \mathbf{3 0 E}=30 \mathrm{t}$ de $\mathrm{E} \mathrm{ha}^{-1} ; \mathbf{4 5 E}=45 \mathrm{t}$ de $\mathrm{E} \mathrm{ha}{ }^{-1}$;

$\mathbf{E M = E ~ m a ́ s ~ M ; ~ F Q = F e r t i l i z a c i o ́ n ~ Q u i ́ m i c a ~ ( 1 6 0 - 6 0 - 3 0 ~} \mathrm{kg} \mathrm{ha}^{-1}$ de NPK);

$\mathbf{P V}=$ primavera-verano; $\mathbf{O I}=$ Otoño-Invierno. 

productivo de maíz forrajero (Zea mays L.)

En OI, se registró el mayor contenido de $\mathrm{N}$ en 15EM y 45 EM con respecto al resto de los tratamientos $(P<0.05)$. El contenido de $\mathrm{N}$ en suelo fue mayor en todos los tratamientos durante el OI con relación a los mismos tratamientos en PV $(P<0.05)$ (Fig. 3A). El contenido de $\mathrm{P}$ en suelo durante el OI fue mayor en los tratamientos en donde se aplicó EC con o sin M con relación a SF y FQ $(P<0.05)$. En PV, 15E, 30E, 15EM y 45EM aumentaron $(P<0.05)$ el $\mathrm{P}$ en suelo con respecto a los mismos tratamientos en OI (Fig. 2) (Fig. 3B). El K en suelo fue mayor en $45 \mathrm{E}$ y 45 EM durante PV y OI con relación a SF y FQ en ambos ciclos $(P<0.05)$ (Fig. 3C). El Ca en suelo fue mayor en PV en los tratamientos estudiados con respecto a los mismos tratamientos en OI $(P<0.05)$ (Fig. 3D).

El CIC fue afectado por el tratamiento y el ciclo $(P<0.05)$. Las medías de cuadrados mínimos para los tratamientos se observan en la Fig. 4. En PV, el CIC fue mayor al de OI, 15.8 y $14.1^{\mathrm{b}}$, respectivamente.

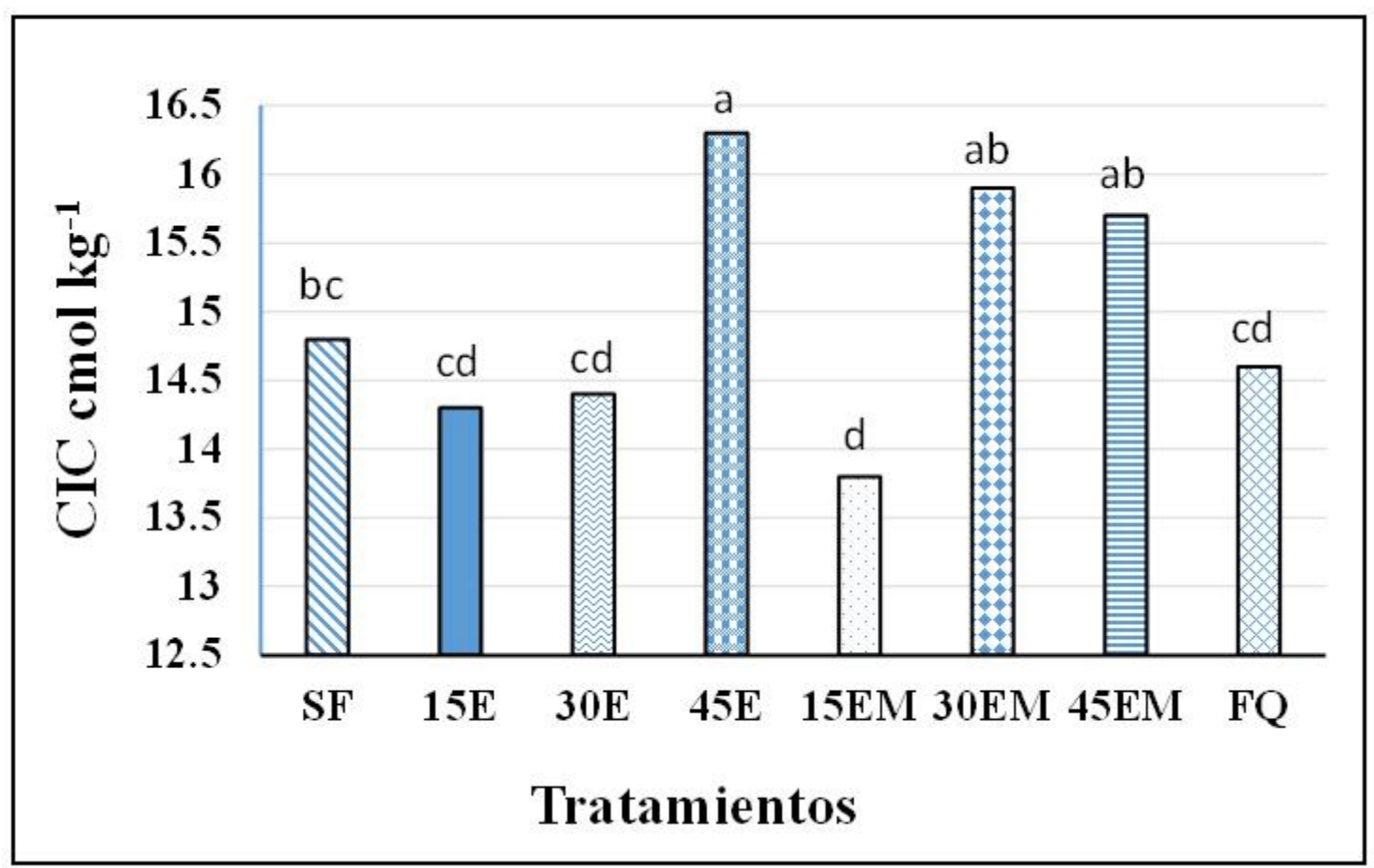

Fig. 4. Efecto de la fertilización con estiércol composteado (E) y el hongo micorrízico arbuscular (M), Glomus intraradices en maíz forrajero sobre la capacidad de intercambio catiónico (CIC) en el suelo. Literales distintas entre tratamientos indican diferencia $(P<0.05) . \mathbf{S F}=$ Sin Fertilizar; 15E=15 $\mathrm{t}$ de $\mathrm{E} \mathrm{ha}^{-1} ; \mathbf{3 0 E}=30 \mathrm{t}$ de $\mathrm{E} \mathrm{ha}^{-1} ; \mathbf{4 5 E}=45 \mathrm{t}$ de $\mathrm{E}$ ha $^{-1} ; \mathbf{E M}=\mathrm{E}$ más $\mathrm{M}$; FQ=Fertilización Química (160-60-30 kg ha ${ }^{-1}$ de NPK). 


\section{Porcentaje de infección de $M$ en raíz y características agronómicas de la planta}

El porcentaje de infección de $\mathrm{M}$ en raíz no fue afectado por el tratamiento y por la interacción tratamiento $\mathrm{x}$ ciclo $(P>0.05)$. En el ciclo $\mathrm{PV}$ el porcentaje de infección de $\mathrm{M}$ en raíz fue mayor al de OI, $28.0^{\mathrm{a}} \pm 2.9$ y $18.5^{\mathrm{b}} \pm 2.0$, respectivamente. La altura y número de hojas de las plantas fue mayor en todos los tratamientos del ciclo PV con respecto a OI $(P<0.05)$. En 30EM y FQ durante el ciclo PV se detectó la mayor altura de las plantas con relación al resto de los tratamientos $(P<0.05)$. La MS de la planta fue mayor $(P<0.05)$ en $15 \mathrm{E}, 15 \mathrm{EM}, 45 \mathrm{EM}$ y FQ durante el ciclo OI con respecto a $\mathrm{PV}$.

\section{Índice de crecimiento en el cultivo, acumulación de nutrientes y rendimiento productivo}

A los 20 DDS y en PV, 30EM y FQ presentaron el mayor índice de crecimiento con relación al resto de los tratamientos dentro de los mismos DDS $(P<0.05)$. En los 40 DDS y en PV, SF, 15E, $30 \mathrm{E}, 45 \mathrm{E}$ y $15 \mathrm{EM}$ presentaron el mayor índice de crecimiento con relación al resto de los tratamientos dentro de los mismos DDS $(P<0.05)$. Sin embargo, en los 60 DDS y en OI, SF, 15E, $30 \mathrm{E}, 45 \mathrm{E}, 15 \mathrm{EM}$ y $30 \mathrm{EM}$ tuvieron mayor índice de crecimiento con respecto al resto de los tratamientos dentro de los mismos DDS $(P<0.05)$. A los 80 DDS y en OI se registró mayor índice de crecimiento con relación a los mismos tratamientos en PV y dentro de los mismos DDS $(P<0.05)$.

En OI, 30EM presentó la mayor acumulación de $\mathrm{N}$ en planta con respecto al resto de los tratamientos $(P<0.05)$. En PV y OI, SF registro la menor acumulación de $\mathrm{P}$ con relación a los tratamientos con E y E más M $(P<0.05)$. En PV y 45EM se detectó la mayor acumulación de $\mathrm{K}$ $(P<0.05)$. En PV se tuvo mayor acumulación de $\mathrm{Ca}$ en planta en todos los tratamientos con respecto a los mismos tratamientos en $\mathrm{OI}(P<0.05)$.

Con excepción de 45E, el rendimiento de forraje en MV fue mayor en PV en todos los tratamientos con relación a los mismos tratamientos en OI $(P<0.05)$. En ambos ciclos, PV y OI, 30EM tuvo el mayor rendimiento de forraje en MS $(P<0.05)$. 

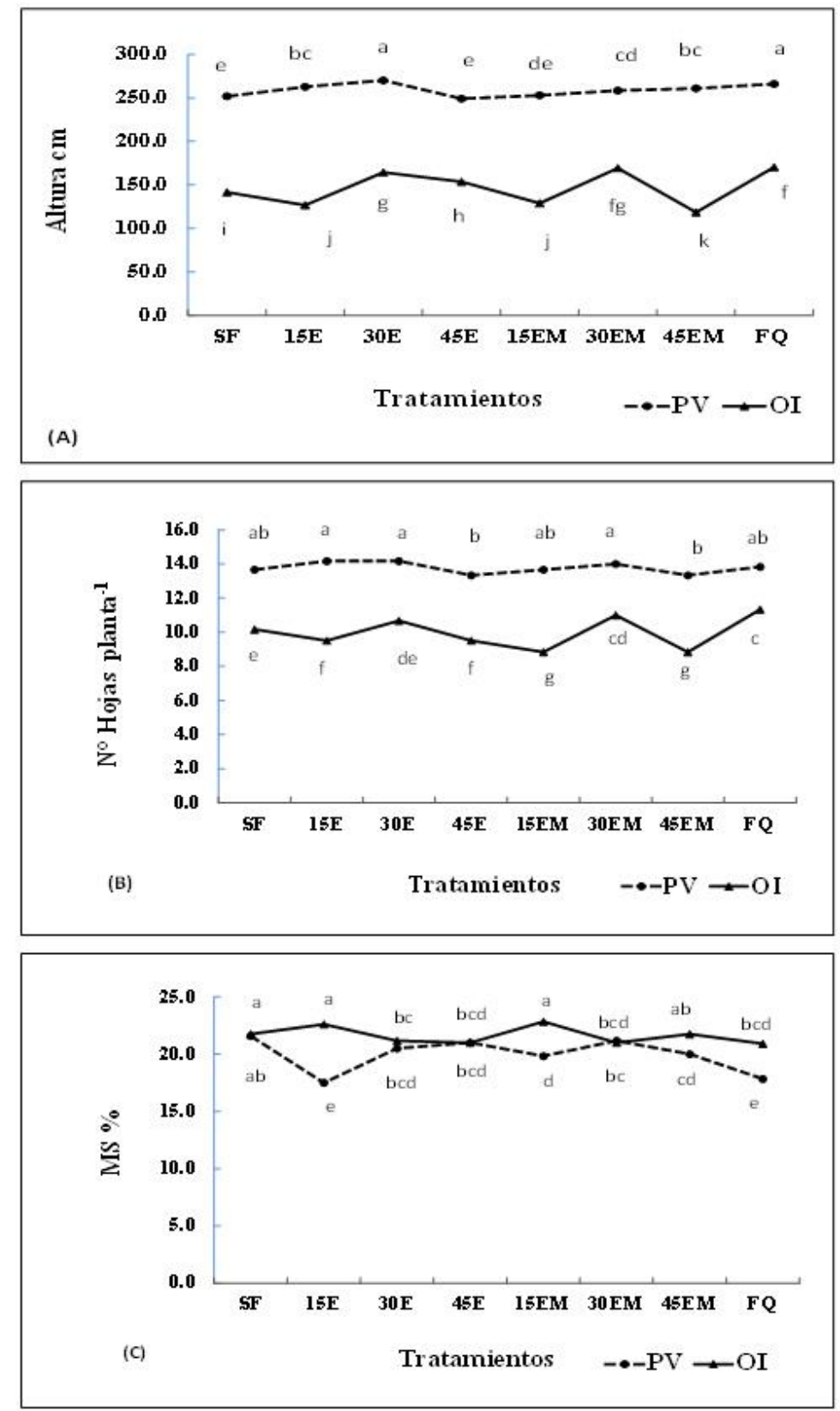

Fig. 5. Respuesta de la fertilización con estiércol composteado (E) y el hongo micorrízico arbuscular (M), Glomus intraradices en maíz forrajero sobre las variables agronómicas altura, número de hojas y MS en dos ciclos de cultivo (PV y OI).

Literales distintas entre ciclo y entre tratamientos indican diferencia $(P<0.05)$. MS=Materia Seca; $\mathbf{S F}=$ Sin Fertilizar; 15E $=15 \mathrm{t}$ de $\mathrm{E} \mathrm{ha}^{-1} ; \mathbf{3 0 E}=30 \mathrm{t}$ de $\mathrm{E} \mathrm{ha}^{-1} ; \mathbf{4 5 E}=45 \mathrm{t}$ de $\mathrm{E} \mathrm{ha}^{-1}$; $\mathbf{E M}=$ E más M; FQ=Fertilización Química (160-60-30 kg ha-1 de NPK); PV=PrimaveraVerano; OI=Otoño-Invierno. 


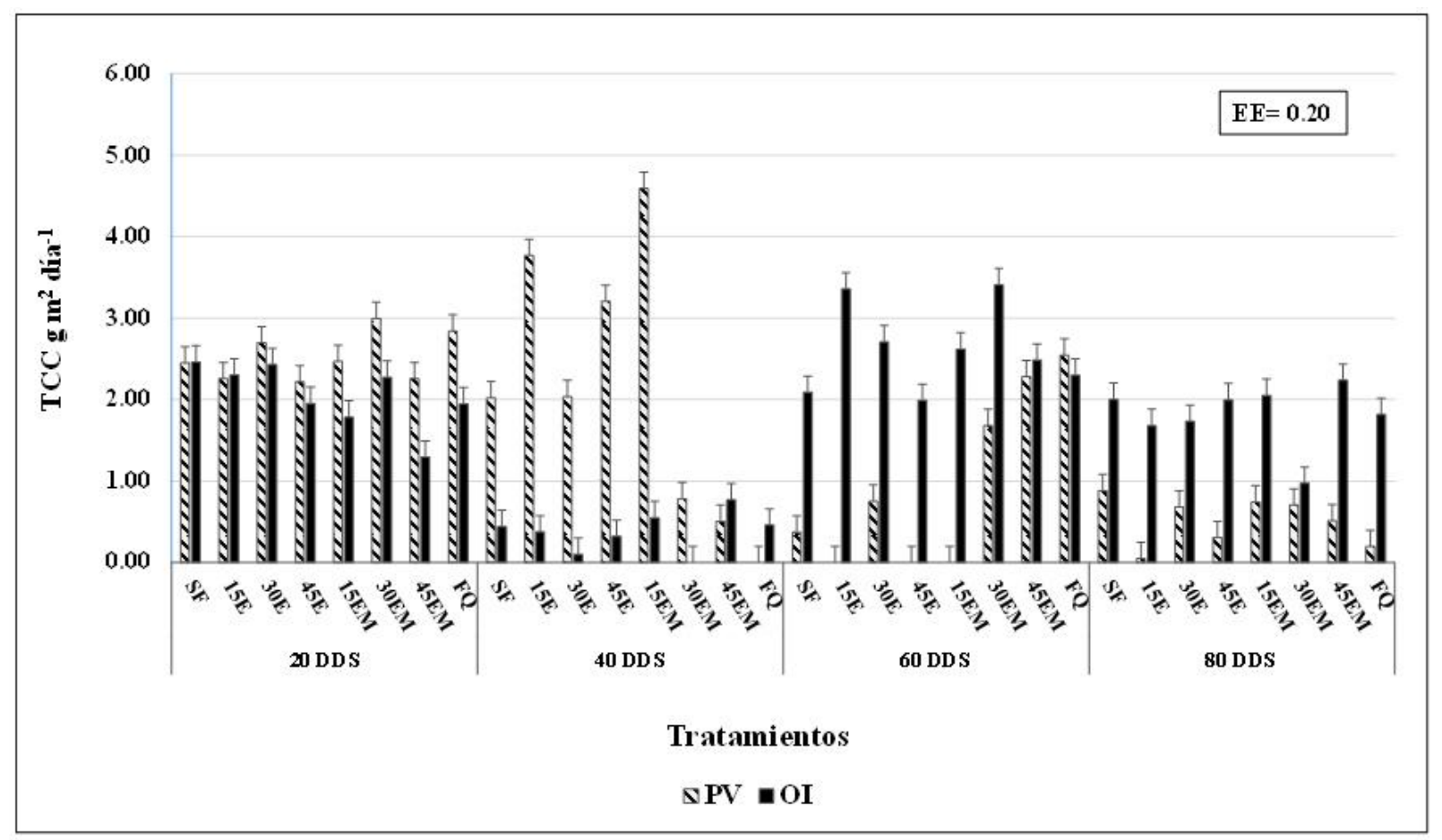

Fig. 6. Comportamiento de la tasa de crecimiento del cultivo de maíz (TCC) a los 20, 40, 60 y 80 días después de la siembra (DDS), empleando estiércol composteado (E) y el hongo micorrízico arbuscular (M), Glomus Intraradices en dos ciclos de cultivo (PV y OI). EE=Error Estándar; $\mathbf{S F}=$ Sin Fertilizar; 15E=15 t de $E$ ha $^{-1} ; \mathbf{3 0 E}=30 \mathrm{t}$ de E ha $^{-1} ; \mathbf{4 5 E}=45$ t de E ha ${ }^{-1} ; \mathbf{E M = E ~ m a ́ s ~ M ; ~ F Q = F e r t i l i z a c i o ́ n ~ Q u i ́ m i c a ~ ( 1 6 0 - 6 0 - 3 0 ~ k g ~}$ ha $^{-1}$ de NPK); PV=Primavera-Verano; OI=Otoño-Invierno. 

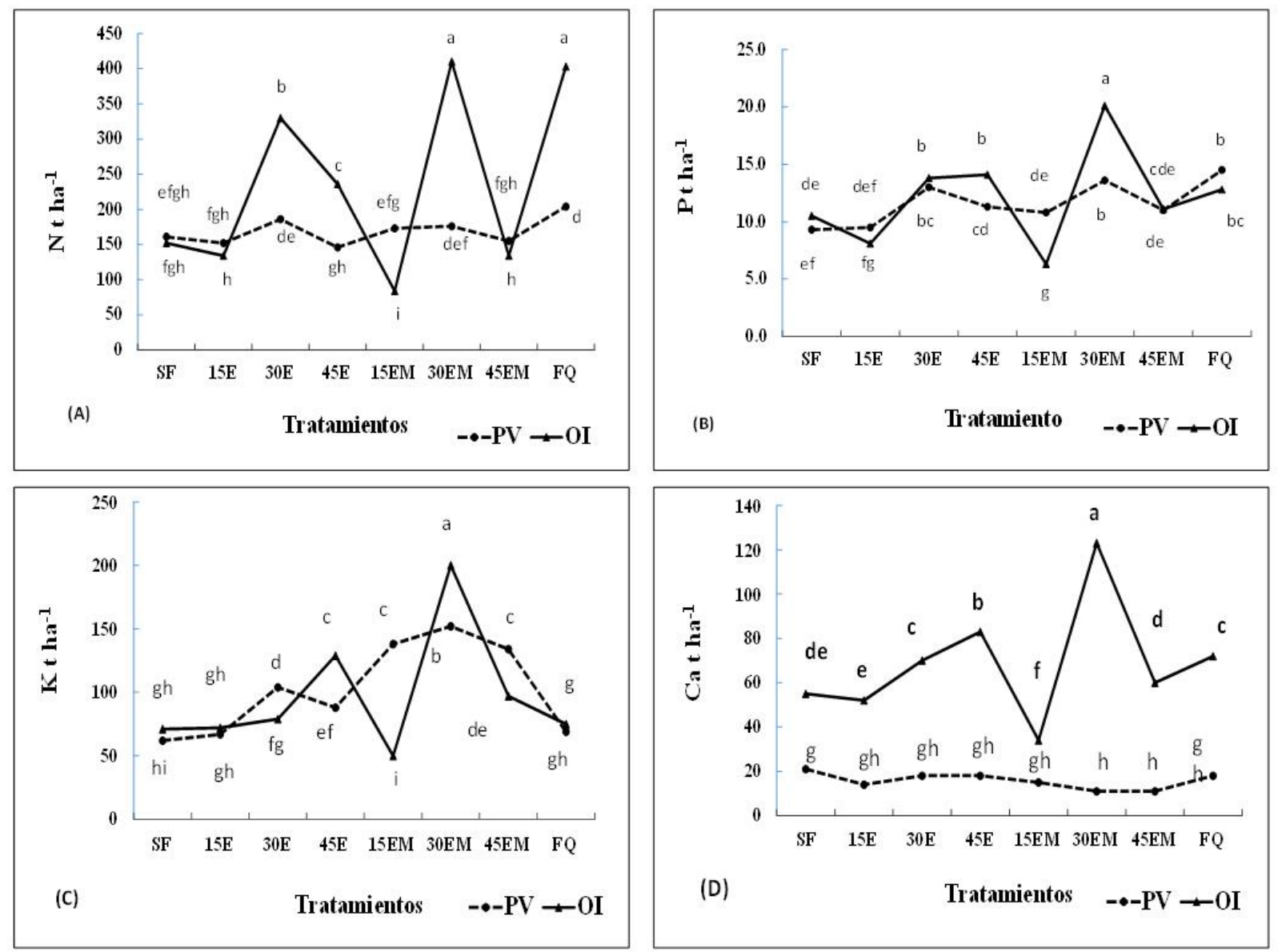

Fig. 7. Acumulación de nutrientes en el cultivo de maíz forrajero en respuesta a la fertilización con estiércol composteado (E) y el hongo micorrízico arbuscular (M),

Glomus intraradices en dos ciclos de cultivo (PV y OI).

Literales distintas entre ciclo y entre tratamientos indican diferencia $(P<0.05)$. $\mathbf{S F}=$ Sin Fertilizar; 15E $=15 \mathrm{t}$ de $\mathrm{E} \mathrm{ha}^{-1} ; \mathbf{3 0 E}=30 \mathrm{t}$ de $\mathrm{E} \mathrm{ha}^{-1} ; \mathbf{4 5 E}=45 \mathrm{t}$ de $\mathrm{E} \mathrm{ha}^{-1}$;

$\mathbf{E M = E ~ m a ́ s ~ M ; ~ F Q = F e r t i l i z a c i o ́ n ~ Q u i ́ m i c a ~ ( 1 6 0 - 6 0 - 3 0 ~} \mathrm{kg} \mathrm{ha}^{-1}$ de NPK); $\mathbf{P V}=$ Primavera-Verano; $\mathbf{O I}=$ Otoño-Invierno. 

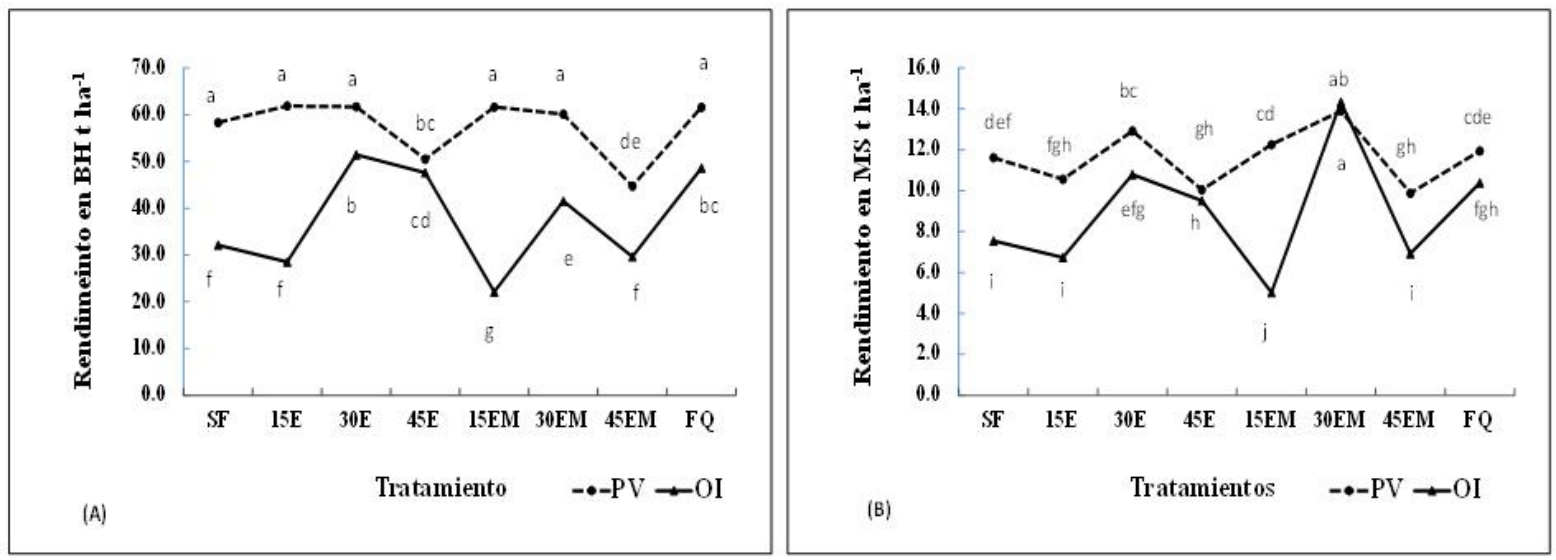

Fig. 8. Efecto de la fertilización con estiércol composteado (E) y el hongo micorrízico arbuscular (M), Glomus intraradice en maíz forrajero sobre el rendimiento total en materia verde (MV) y materia seca (MS) en dos ciclos de cultivo (PV y OI).

Literales distintas entre ciclo y entre tratamientos indican diferencia $(P<0.05)$. SF $=$ Sin Fertilizar; 15E $=15 \mathrm{t}$ de $\mathrm{E}$ ha ${ }^{-1} ; \mathbf{3 0 E}=30 \mathrm{t}$ de $\mathrm{E} \mathrm{ha}^{-1} ; \mathbf{4 5 E}=45 \mathrm{t}$ de $\mathrm{E} \mathrm{ha}^{-1} ; \mathbf{E M}=\mathrm{E}$ más $\mathrm{M}$;

FQ=Fertilización Química (160-60-30 kg ha-1 de NPK); PV=Primavera-Verano;

OI=Otoño-Invierno.

\section{Discusión}

La PP acumulada y la temperatura ambiente registradas durante el ciclo PV fueron mayores a la registrada en OI. En ambos ciclos, la PP y la temperatura ambiente permitieron el crecimiento adecuado del cultivo de maíz, debido a que una PP acumulada entre 500 y $1000 \mathrm{~mm}$ es considerada como óptima para el desarrollo del cultivo, así como temperaturas entre 20 y $30{ }^{\circ} \mathrm{C}$ (Tinoco et al., 2002, 8). La mayor PP y temperatura ambiente que se presentó en PV contribuye a explicar las diferencias a favor de la altura, número de hojas y rendimiento forrajero en MS de las plantas que crecieron durante este ciclo con respecto a las de OI. Respecto a los nutrientes reportados en el E se observa un elevado nivel de $\mathrm{MO}$, lo cual puede atribuirse al origen del material composteado ( $100 \%$ estiércol de bovinos en pastoreo). El Nt se encuentra dentro de los límites reportados, ya que los contenidos de Nt en las compostas pueden ir de 0.3 a $1.5 \%$ dependiendo del material de origen (FAO, 2013, 36). Incluso se ha mencionado que el Nt en un abono orgánico no debe de exceder del 2\% (Castro et al., 2009, 35). El suelo presentó 0.22\% de $\mathrm{Nt}$, valores medios de $\mathrm{P}$ y $\mathrm{Ca}$, y de acuerdo a su CIC se le puede considerar como un suelo con una reserva nutrimental media (SEMARNAT, 2002, 29). 
Influencia del estiércol composteado y micorriza arbuscular sobre la composición química del suelo y el rendimiento productivo de maíz forrajero (Zea mays L.)

\section{Composición química del suelo y número de esporas}

En el primer ciclo PV, la aplicación de E con o sin M aumento el pH en suelo con relación al tratamiento SF. Sin embargo, en el segundo ciclo OI, el pH no se afectó en la misma magnitud como en el primer ciclo. De acuerdo con Osorio $(2012,3)$, el pH puede indicar que hay disponibilidad o limitación de nutrientes en el suelo. A pH menores de 5.5 se restringe la solubilidad y/o disponibilidad de algunos nutrientes. Esto debido a la presencia de iones de aluminio, los cuales determinan la solubilidad del fosfato, hierro, manganeso, entre otros, o escasez de calcio, magnesio, potasio, sodio en sus formas disponibles. Al parecer, la aplicación de $\mathrm{E}$ con o sin $\mathrm{M}$ durante el primer ciclo favoreció la disponibilidad de nutrientes por permitir una reducción en el pH del suelo lo que contribuye a explicar el mayor rendimiento forrajero en MS que se presentó durante la PV. Por otro lado, cuando se aplica composta de estiércol combinado con un fertilizante mineral en suelos franco-arcilloso-arenoso, el $\mathrm{pH}$ del suelo puede aumentar significativamente (Gil et al., 2008, 1438). En el segundo ciclo OI, no se detectaron cambios a favor del uso de $\mathrm{E}$ con o sin $\mathrm{M}$. Al respecto, una disminución del $\mathrm{pH}$ en el suelo puede deberse a más factores como la descomposición de la MO. En el proceso de descomposición de la $\mathrm{MO}$, la respiración de los microorganismos produce ácidos orgánicos y $\mathrm{CO}_{2}$, el cual forma ácido carbónico (Osorio, 2012, 3). Además, el contenido de $\mathrm{N}$ en la MO inicial también modifica el pH aumentándolo inicialmente por el consumo de protones para la producción de amonio $\left(\mathrm{NH}_{4}{ }^{+}\right)$, y disminuyéndolo seguidamente por la liberación de protones a la solución del suelo debido a la nitrificación del amonio $\left(\mathrm{NH}_{4}{ }^{+}\right)$a nitrato $\left(\mathrm{NO}_{3}^{-}\right)$(Martínez et al., 2008, 73). Este tipo de cambios en la $\mathrm{MO}$ y en el $\mathrm{N}$ de la $\mathrm{MO}$ pueden explicar la ausencia de cambios favorables en el $\mathrm{pH}$ atribuible al uso de $\mathrm{E}$ con o sin $\mathrm{M}$.

Con respecto a la FQ, la fertilización mineral influye en la disminución del pH del suelo después de continuas aplicaciones (Gil et al., 2008, 1438; Adebayo et al., 2018, 71) y en un manejo convencional con monocultivos se propicia una disminución del $8.4 \%$ del valor del $\mathrm{pH}$ (Palma et al., 2007, 95). Sin embargo, los resultados del presente estudio no corroboran la disminución del $\mathrm{pH}$ en el tipo de suelo estudiado durante dos ciclos de cultivo subsecuentes.

Una de las desventajas que pudieran tener los abonos orgánicos es la cantidad de sales que pueden contener, las cuales se van acumulando en el suelo a través de la constante aplicación de éstos (Quiroga, et al., 2011, 202). La aplicación de 45E y EM incremento la CE durante el primer ciclo PV. Sin embargo, en el segundo ciclo OI, se redujo la influencia del E con o sin M sobre la 
CE. Lo anterior puede atribuirse a la eliminación de sales por lavado natural del suelo debido a las PP presentadas en ambos ciclos de cultivo (Fig.1). En apoyo a lo detectado en el presente estudio, la aplicación de diferentes dosis de estiércol para la producción de forraje de maíz en una zona seca desértica, no afecto la CE en el primer año de aplicación, la cual tuvo valores menores a $2 \mathrm{dS} \mathrm{m}^{-1}$ (Trejo et al., 2013, 734). Sin embargo, en otros estudios, la aplicación de estiércol de borrego en suelos con enmiendas orgánicas comerciales, se incrementó la CE desde 0.5 hasta 1.6 dS m m $^{-1}$ año $^{-1}$ (Álvarez et al., 2006, 267). En el primer ciclo PV, el número de esporas fue mayor en PV con relación al resto de los tratamientos. En el segundo ciclo OI, se incrementó el número de esporas en suelo en SF, FQ y los tratamientos E sin M con relación a lo detectado en el primer ciclo PV. Al respecto, se reporta que un incremento de la CE aumenta la concentración de sales en el suelo, y disminuye su contenido de agua. Esta condición se refleja en una disminución del potencial osmótico (concentración real de sal en el suelo) afectando a los microrganismos del suelo y por consecuencia la mineralización de la MO (Setia et al., 2011, 1909). En este sentido, la ausencia de cambio y una disminución de la CE en suelo durante el segundo ciclo OI pudo favorecer el incremento en el número de esporas en SF, FQ y E sin M.

En investigaciones realizados por Setia et al. $(2011,1912)$, quienes manejaron diferentes niveles de $\mathrm{CE}$, encontraron que con niveles de $5 \mathrm{dS} \mathrm{m}{ }^{-1}$, disminuye el $\mathrm{C}-\mathrm{CO}_{2}$ acumulado, así como también la descomposición del carbono orgánico particulado a través del tiempo; esto debido a que los microorganismos que intervienen en la descomposición temprana de la MO se ven afectados por la salinidad. Yan et al. (2015, 319), también documentó que la respiración del suelo disminuye más del $50 \%$ cuando la $\mathrm{CE}$ es $\geq$ a $5 \mathrm{dS} \mathrm{m}^{-1}$, debido a que la acumulación de sales en el suelo disminuye la biomasa microbiana primariamente porque el estrés osmótico induce el secado y la lisis de las células.

Aunque la CE fue afectada por los diferentes tratamientos y entre ciclo en el presente estudio, los valores detectados al final del estudio fueron entre 0.032 y $0.143 \mathrm{dS} \mathrm{m}^{-1}$, lo cual indica una baja concentración salina en el suelo. Los bajos valores de CE en los tratamientos orgánicos empleados garantizan que los niveles de sales en el suelo $\left(>4 \mathrm{dS} \mathrm{m}^{-1}\right)$ no afecte el crecimiento adecuado de las plantas en el cultivo; La acumulación de osmolitos en el suelo (sales inorgánicas), deriva en un pobre crecimiento de las plantas y microorganismos. Por un lado, el alto potencial osmótico en la solución del suelo genera sequedad fisiológica en la planta ocasionando su muerte. Las plantas que puedan adaptarse a un bajo potencial osmótico, 
demeritan en su desarrollo debido a que la síntesis de osmolitos demanda grandes cantidades de energía no siendo suficiente ésta para el cultivo (Yan et al., 2015, 318).

Con relación al tratamiento $\mathrm{FQ}$, la aplicación de $\mathrm{E}$ con o sin $\mathrm{M}$ incremento la $\mathrm{MO}$ en suelo, pero, este aumento en MO no se detectó con relación al tratamiento SF. La incorporación al suelo de estiércol composteado combinado con fertilizante mineral incrementa la $\mathrm{MO}$ en suelo (Gil et al., 2008, 1438), lo que apoya lo registrado en el presente estudio. A diferencia de los resultados indicados en este estudio, evaluaciones realizadas con diferentes dosis de estiércol indican un aumento en la $\mathrm{MO}$ desde el primer año de aplicación respecto al nivel inicial (Trejo et al., 2013, 731). Sin embargo, en zonas áridas el aumento de la MO puede implicar la aplicación de estiércol por periodos de tiempo más largos, como lo reporta Salazar et al. (2010, 384), con un trabajo de seis años de aplicación de $40 \mathrm{t}$ estiércol ha ${ }^{-1}$ el contenido de MO aumento de 0.99 a $2.7 \%$.

Aunque, existen evidencias que indican que la aplicación de composta tiene un efecto positivo sobre el contenido de $\mathrm{MO}$ en el suelo como se mencionó previamente, también se ha indicado ausencia de influencia del uso de composta en un periodo corto, por lo que es necesario evaluar el uso de esta enmienda orgánica después de un año de aplicación continua bajo estas condiciones como lo sugieren Gil et al. $(2008,1438)$. La ausencia de influencia de la aplicación de $\mathrm{E}$ con o sin M sobre la MO en suelo puede ser atribuida a la humedad y temperatura del suelo, factores que influyen en la rápida mineralización de la MO (Zech et al., 1997, 133).

Los resultados encontrados coinciden con lo indicado en un estudio realizado en una zona seca desértica de suelos francos y arcillosos, en donde la aplicación de 30 t de estiércol bovino ha $^{-1}$ incrementó los niveles de N y P en 185 y $310 \%$, respectivamente. Sin embargo, se reporta poca influencia sobre el contenido de Ca, Mg, Na y K (López et al., 2001, 299). Las enmiendas orgánicas en combinación que fertilizantes químicos permiten también el incremento en la calidad del suelo a través del tiempo. En suelo de llanura de río la aplicación de 40 t ha ${ }^{-1}$ de estiércol de granja año ${ }^{-1}$ más $100 \mathrm{~kg} \mathrm{~N} \mathrm{ha}^{-1}$ año $^{-1}$ a suelo cultivado con maíz para ensilaje, el nivel de Nt aumento en 46.7\% en 11 años bajo este manejo (Monaco et al., 2008, 611). Bajo diferentes condiciones de suelo y ambiental la incorporación de enmiendas orgánicas solas o combinadas con fertilizantes sintéticos, logran cambiar los niveles de nutrientes en el suelo en diferentes proporciones. 
La incorporación del $\mathrm{M}$ en el presente estudio no refleja un aumento de esporas en los tratamientos EM respecto a SF y a los tratamientos sin M, tanto en PV como en OI, esto puede indicar la presencia de poblaciones de esporas de $\mathrm{M}$ en la zona de estudio. Entre ciclos, se observa más esporas en OI que en PV, probablemente a que una menor temperatura ambiente en OI con relación a PV influye en la actividad fisiológica de algunas especies de M, propiciando la esporulación (Barrer, 2009, 128). Sin embargo, el uso de biofertilizantes en los sistemas agroecológicos permite la permanencia de estos organismos benéficos, quienes movilizan y reciclan nutrientes que son aprovechados por las plantas, además que ayudan a la estructura del suelo a través de sus hifas que permiten una mejor aireación y distribución de la humedad (Barrer, 2009, 124). Aunque la influencia del M empleado no se reflejó en un incremento en el número de esporas, su incorporación en el nivel 30EM permitió mayor acumulación de $\mathrm{N}, \mathrm{P}, \mathrm{K}$ en planta, así como mayor rendimiento productivo de forraje en BS con relación a los tratamientos SF y FQ.

\section{Porcentaje de infección de $M$ en raíz y características agronómicas de la planta}

A pesar de que el número de esporas se favorecido en OI, el mayor porcentaje de infección en raíz se registró para las plantas crecidas durante el ciclo PV. Estos resultados coinciden con el reportado por Pérez $(2012,56)$, quien evaluó el uso de vermicomposta en el cultivo de maíz, no encontrando diferencia estadística en el porcentaje de infección de raíz entre vermicomposta y cuando lo combino con M Glomus. La ausencia de influencia de los tratamientos evaluados sobre el porcentaje de infección en la raíz puede deberse a que la evaluación del cultivo se realizó en la etapa de la cosecha, y se han reportado mayores valores de infección al inicio de fructificación de las plantas que en la etapa de cosecha (Martín y Rivera, 2015, 41; Montaño et al., 2001, 341).

La aplicación de E con y sin M tuvo una respuesta variable en la altura, número de hojas y MS de la planta. Al respecto, existen evidencias que indican que el uso del estiércol en el cultivo de maíz forrajero, no afecta la altura y MS respecto a la fertilización inorgánica y al testigo (López-Calderón et al., 2015, 11). Sin embargo, el uso de 40 t de estiércol ha ${ }^{-1}$ durante seis años, incrementó la altura del maíz respecto a la fertilización química con valores de 262.8 y $255.1 \mathrm{~cm}$, respectivamente (Salazar et al., 2010, 384). Las mayores alturas de la planta y número de hojas de las plantas se presentaron en PV, periodo en que se presentaron condiciones climáticas favorables para el crecimiento de las plantas. Se observa también que la altura, número de hojas y 
MS registradas con las dosis de EC tuvieron respuesta similar a la fertilización química en ambos ciclos de cultivo. Estos valores concuerdan con los obtenidos por Barrón et al. (2014, 314) reportando promedios de $264 \mathrm{~cm}$ y $22.7 \%$ para altura y MS, respectivamente, para la misma zona de estudio, pero con fertilización química. Los resultados obtenidos con E con y sin M indican que el aporte de nutrientes por parte del E permite obtener una altura y número de hojas similar a la obtenida con FQ desde la primera aplicación y que puede sustituir el uso de fertilizante químico.

\section{Tasa de crecimiento en el cultivo, acumulación de nutrientes y rendimiento productivo}

El uso de la fertilización con E y M favoreció el desarrollo del cultivo de maíz forrajero. En general el crecimiento del cultivo fue superior en al menos un tratamiento orgánico respecto al tratamiento FQ en las cuatro edades de evaluación. El desarrollo del cultivo en su etapa inicial, los primeros 20 DDS tuvo menor variación por influencia de los tratamientos y ciclos estudiados con relación a los otros DDS. Este tipo de respuesta puede atribuirse a la ausencia de condiciones extremas en PP y temperatura ambiente, situación que favoreció el uso de los nutrientes disponibles para cada uno de los tratamientos estudiados. Sin embargo, a los 40 DDS del ciclo OI la TCC fue bajo, lo que puede deberse a la reducción de temperatura ambiente que se presentó en esta etapa de desarrollo de la planta (Fig. 1). Además, parte del ciclo OI se desarrolló durante la época de nortes, la cual se caracteriza por días nublados y lluvias intermitentes. El crecimiento a los 60 y 80 DDS en PV fue menor al detectado en OI, este tipo de respuesta puede atribuirse a ocurrencia del periodo canicular. La canícula se caracteriza por altas temperaturas y baja precipitación pluvial, presentándose entre julio y agosto. Aunado a las condiciones ambientales, el crecimiento del cultivo se da en forma acelerada al inicio, con la formación de hojas, y antes de la floración. Posteriormente, los nutrientes se utilizan para el desarrollo de espigas y fructificación, por lo que el aumento en la biomasa disminuye.

La mayor TCC se presentó en PV durante los dos primeros periodos de crecimiento. El aprovechamiento de los nutrientes proporcionados en los diferentes niveles de E se reflejó con la mayor producción de hojas y las mayores alturas registradas en ese ciclo. En ese mismo ciclo, PV, la TCC disminuyó a los 80 DDS ya que el destino de los nutrientes disponibles en el suelo se orienta hacia el desarrollo del fruto más que al aumento de la biomasa. 
En los sistemas de producción de rumiantes domésticos, es importante obtener forrajes con alto valor nutritivo, con el fin de cubrir el mayor porcentaje de nutrimentos que demandan los animales en cada una de sus etapas fisiológicas y productivas (Enríquez et al., 2011, 277). En este sentido, la incorporación de E y M al suelo permitió que las plantas acumularan una mayor cantidad de nutrientes. Un mayor acumulamiento de $\mathrm{Nt}$, al encontrado en el presente trabajo, se reporta con la aplicación de 80 t de estiércol ha ${ }^{-1}$, estiércol + fertilizante mineral y composta de estiércol + fertilizante mineral; la acumulación de $\mathrm{N}$ por el cultivo fue de 253,223 y $249 \mathrm{~kg} \mathrm{ha}^{-1}$ en el año de aplicación respectivamente, sin embargo, no encontraron diferencias significativas entre los tratamientos evaluados (Figueroa et al., 2010, 366). Los resultados del presente estudio indican que los beneficios en la acumulación de Nt en planta como consecuencia de aplicar E con y sin M se manifiestan con mayor énfasis en el segundo ciclo, OI, y la acumulación de Nt en planta es mayor en estos tratamientos con relación SF, pero, similar a FQ. Sin embargo, en otros estudios efectuados con diferentes niveles de estiércol aplicados al cultivo de maíz, los tratamientos orgánicos permitieron una mayor extracción de $\mathrm{N}, \mathrm{P}, \mathrm{K}$ y $\mathrm{Ca}$ por las plantas en comparación a la fertilización mineral y al testigo (Salazar et al., 2007, 181). Mientras que, en maíz forrajero, se tuvo una extracción menor a $200 \mathrm{~kg}_{\text {de }} \mathrm{N} \mathrm{ha}^{-1}$ cuando se incorporó al suelo estiércol y composta de estiércol, comparada con fertilización mineral (Ferguson et al., 2005, 1676).

Una de las desventajas del uso abonos orgánicos es la lenta mineralización que puede ir de semanas a meses, por lo que la disponibilidad de nutrientes en el suelo no suele ser rápida (Castro et al., 2009, 32). Lo que explica, en parte, la mayor acumulación de $\mathrm{N}$ y Ca en planta en el segundo ciclo con respecto al primer ciclo. Además, en su conjunto se pueden presentar pérdidas de nutrientes de los agroecosistemas a través de la desnitrificación del suelo, escorrentía superficial, volatilización y lixiviación (Shanahan et al., 2008, 52). Por lo demás, es importante considerar que el uso del E más M no favoreció una mayor cantidad de esporas en suelo ni aumento el porcentaje de infección en raíz. Sin embargo, permitió una mayor acumulación de nutrientes, lo que confirma el papel que desempeñan las micorrizas en la captura de nutrientes del suelo a través de sus hifas que abarcan mayor superficie. Si bien se ha puntualizado que los $\mathrm{M}$ favorecen la extracción de $\mathrm{P}$ en suelos deficientes, también benefician la fijación de $\mathrm{N}$, la absorción de agua y mejora la eficiencia de uso de la planta de los nutrientes (Barrer, 2009, 124). 
Los datos obtenidos respecto a la acumulación de $\mathrm{P}$ en el presente trabajo, son mayores (20 $\mathrm{t} \mathrm{ha}^{-1}$ en 30EM) comparados con datos reportados en otros trabajos donde obtuvieron solo 0.512 t $\mathrm{P} \mathrm{ha}^{-1}$ al aplicar $40 \mathrm{t}$ de estiércol ha-1 (Salazar et al., 2007, 182). Las evaluaciones realizadas por Ferguson et al. $(2005,1677)$ muestran que la absorción de P por el cultivo no presentó diferencia significativa entre los tratamientos orgánicos evaluados en años individuales; sin embargo, el valor promedio registrado al primer año, la fertilización inorgánica (33 kg de P $\mathrm{ha}^{-1}$ ) fue significativamente menor a la fertilización orgánica $\left(37.5 \mathrm{~kg}\right.$ de $\left.\mathrm{P} \mathrm{ha}^{-1}\right)$. Valores similares a los obtenidos en el presente trabajo, son los reportados por Pérez $(2012,56)$ cuando evaluó el uso del humus de lombriz con Glomus y Azospirillum en el cultivo de maíz; valores de 9.8 y $11.8 \mathrm{~kg}$ de $\mathrm{P} \mathrm{ha}^{-1}$ en el forraje de maíz fueron obtenidos en los tratamientos de Humus + Glomus y Humus + Glomus + Azospirillum, respectivamente, los cuales no mostraron diferencia significativa entre ellos, pero si la combinación de Humus + Glomus + Azospirillum $(P<0.05)$ con respecto al testigo con $4.7 \mathrm{~kg}$ de $\mathrm{P} \mathrm{ha}^{-1}$ en el forraje de maíz. De acuerdo los valores obtenidos en la acumulación de nutrientes por el cultivo, se observó que el nutriente que más se acumuló fue el $\mathrm{N}$ en comparación al resto de los nutrientes. Esto es debido a que el $\mathrm{N}$ es el nutriente más demandado por la planta para su desarrollo (Trejo et al., 2013, 728). Debido a esto, generalmente la aplicación de un abono orgánico se estima más por las necesidades de N, no considerando la cantidad de $\mathrm{P}$ que aporta el abono el cual resulta luego en un excedente en el suelo (Diacono y Montemurro, 2010, 414).

El E es una fuente de MO que proporciona elementos como sustancias húmicas, su aplicación al suelo junto con el uso del M proporciona una respuesta positiva tanto en el crecimiento como en la calidad nutritiva del forraje. Los componentes principales de la MO la cual es aportada por el E son: carbohidratos, proteínas, lípidos y lignina, este último componente y sus productos de la degradación, son precursores de los compuestos aromáticos de carbono a sustancias húmicas (García et al., 2005, 127). Los ácidos húmicos estimulan la síntesis de H+-ATPasa, la cual es necesaria para las membranas plasmáticas de las hifas de la micorriza. La micorriza interviene en algunos aspectos fisiológicos de las plantas como la fotosíntesis debido a un aumento en la asimilación de $\mathrm{CO}_{2}$, consecuencia de una mayor absorción de agua y nutrientes. También interviene en la producción de hormonas aumentando la citosina en la raíz, lo que induce a la división celular y por ende el crecimiento de la planta (Pimienta et al., 2009, 71). Un mayor crecimiento de hojas es mayor disponibilidad de área fotosintéticamente activa. La captación de nutrientes en la micorriza se da a través de la absorción de nutrientes por el micelio fúngico, la translocación de los nutrientes de las 
estructuras del hongo hacia la raíz de la planta, y por último la transferencia de nutrientes a las células de la planta vía interfaces simbiótica. De igual forma la transferencia de las hexosas de la planta al hongo es por medio de interfaces. Las interfaces pueden ser intercelulares (las hifas crecen en los espacios intercelulares de la superficie de la raíz) o intracelulares (los arbúsculos crecen dentro de la célula) (Smith y Read, 2008, 122).

Lo anterior puede verse reflejado tanto en la acumulación de nutrientes como en el rendimiento total del cultivo, ya que los valores más altos fueron para el tratamiento 30EM. El rendimiento de forraje en MV de 15EM y 30EM durante PV fue fueron iguales a FQ, y 30E igual a FQ en OI, y el mayor rendimiento total en MS se obtuvo con 30EM en ambos ciclos. En estudios realizados con híbridos de maíz para forraje y fertilización química bajo condiciones agroclimáticas similares a las del presente estudio, los rendimientos promedio de MV y MS fueron de $45.5 \mathrm{t} \mathrm{ha}^{-1}$ y $10.2 \mathrm{t} \mathrm{ha}^{-1}$, respectivamente. Los resultados obtenidos con fertilización orgánica en este estudio, sugieren que se pueden obtener los mismos rendimientos sustituyendo la fertilización química, pero, se debe considerar la concentración de E, el uso de M y el ciclo de cultivo. El rendimiento y composición química del forraje en MS son las variables más importantes al momento de considerar el número de animales que se pueden alimentar con este tipo de forraje (fresco o conservado) puesto que los minerales y la MO presentes en el forraje contribuyen a cubrir los requerimientos de nutrientes para el animal (Enríquez et al., 2011, 116).

\section{Conclusiones}

En las condiciones de suelo y clima que se desarrolló el estudio, la composición química del suelo, acumulación de nutrientes en planta y el rendimiento de maíz forrajero fueron afectados por el uso de estiércol composteado solo o en combinación con $G$. intraradices durante los ciclos PV y OI. La aplicación de $30 \mathrm{t} \mathrm{ha}^{-1}$ de estiércol composteado más G. intraradices fue la mejor opción de fertilización orgánica con relación a sin fertilización y fertilización química debido a que la planta tuvo mayor producción de forraje en MS durante dos ciclos de cultivo sucesivos. El uso de estiércol composteado en combinación de G. intraradices optimizan la utilización de los nutrientes disponibles en el suelo por la planta, situación que favorece la tasa de crecimiento de la planta en sus primeros 20 días de desarrollo y una mayor producción de forraje. La dosis de estiércol composteado con o sin G. intraradices, así como el número de ciclo condicionan la magnitud de la respuesta de tasa de crecimiento, acumulación de nutrientes y 
rendimiento de MS en la planta. El empleo de estiércol composteado con o sin G. intraradices representan una opción para reciclar el estiércol que se genera en las unidades de producción bovina de doble propósito y favorece la sustitución de fertilizantes químicos, así como, el aumento de la calidad química y rendimiento de forraje de maíz, lo que facilita su empleo como alimento para rumiantes sin procesar o para ensilar.

\section{$\underline{\text { Agradecimientos }}$}

La autora principal agradece al Consejo Nacional de Ciencia y Tecnología (CONACYT) por el financiamiento para los estudios de Doctorado en el Programa de Ciencias en Ecología y Desarrollo Sustentable. Al Campo Experimental General Terán, INIFAP, por la donación de la micorriza arbuscular empleada en el trabajo de investigación. Al Ing. Sabel Barrón Freyre (Campo Experimental Huimanguillo, INIFAP) por su asesoría en el establecimiento y desarrollo del cultivo de maíz. Al M en C. Aarón Jarquín Sánchez responsable del laboratorio de Biogeoquímica de El Colegio de la Frontera Sur Unidad Villahermosa, por las facilidades otorgadas para los análisis químicos requeridos.

\section{$\underline{\text { Referencias }}$}

Adebayo, A. O., S. O. Oluremi, y A. O. James, (2018). Influence of compost supplemented with jatropha cake on soil fertility, growth, and yield of maize ( Zea mays $L$.) in a degraded soil of Ilorin, Nigeria. International Journal of Recycling of Organic Waste in Agriculture, 7 (1), 67-73.

Aguilar, C. C., E. J. A. S. Escalante, y M. I. Aguilar (2015). Análisis de crecimiento y rendimiento de maíz en clima cálido en función del genotipo, biofertilizante y nitrógeno. Terra Latinoamericana, 33 (1), 51-62.

Álvarez-Sánchez. E., A. Vázquez- Alarcón, J. Z. Castellanos, y J. Cueto-Wong. (2006). Efectividad biológica de abonos orgánicos en el crecimiento de trigo. Terra Latinoamericana, 24 (2), 261-268.

Averill, C., B. L. Turner, y A. C. Finzi. (2014). Mycorrhiza-mediated competition between plants and decomposers drives soil carbon storage. Nature, 505 (7484), 543-545.

Barrer, S. E. (2009). El uso de hongos micorrizicos arbusculares como una alternativa para la agricultura. Facultad de Ciencias Agropecuarias, 7 (1), 124-132. 
Barrón, F. S., A. M. Barrón, y C. A. Palafox. (2014). Potencial productivo de nueve híbridos de maíz para forraje en condiciones de temporal en la Chontalpa Tabasco. In XXVI Reunión Científica-Tecnológica Forestal y Agropecuária Tabasco y III Simposio Internacional en Producción Agroalimentaria Tropical (pp. 311-316). Villahermosa, Tabasco: Instituto Nacional de Investigaciones Forestales, Agrícolas y Pecuarias.

Castro, A., C. Henríquez, y F. Bertsch. (2009). Capacidad de suministro de N, P y K de cuatro abonos orgánicos. Agronomía Costarricense, 33 (1), 31-43.

Damián-Huato, M. A., B. Ramírez-Valverde, A. Aragón-García, M. Huerta-Lara, D. M de J. Sangerman-Jarquin, y O. Romero-Arenas. (2010). Manejo del maíz en el estado de Tlaxcala, México: entre lo convencional y lo agroecológico. Revista Latinoamericana de Recursos Naturales, 6 (2), 67-76.

Diacono, M., y F. Montemurro. (2010). Long-term effects of organic amendments on soil fertility. A review. Agronomy for Sustainable Development, 30 (2), 401-422.

Díaz, V. T., R. L. Partidas, F. Y. E. Suárez, J. R. Lizárraga, y L. Á. López. (2014). Uso eficiente del agua y producción óptima en maíz, con el uso de cuatro dosis de nitrógeno. Revista Ciencias Técnicas Agropecuarias, 23 (1), 32-36.

Enríquez, Q. J. F., N. F. Meléndez, A. E. D. Bolaños, y E. V. A. Esqueda. (2011). Producción y manejo de forrajes tropicales. (Instituto Nacional de Investigaciones Forestales, Agrícolas y Pecuarias, Ed.) (1ra ed.). Medellín de Bravo, Veracruz, México: Instituto Nacional de Investigaciones Forestales, Agrícolas y Pecuarias.

FAO (Food and Agriculture Organization). (2013). Manual de compostaje del agricultor, Experiencias en América Latina. Oficina Regional de la FAO para América Latina y el Caribe. http://www.fao.org/3/a-i3388s.pdf (31 de marzo de 2017).

Ferguson, R. B., J. A. Nienaber, R. A. Eigenberg, y B. L. Woodbury. (2005). Long-term effects of sustained beef feedlot manure application on soil nutrients, corn silage yield, and nutrient uptake. Journal of Environmental Quality, 34 (sep-oct), 1672-1681.

Figueroa, V. U., W. J. A. Cueto, J. A. Delgado, H. G. Nuñez, S. D. G. Reta, G. H. M. Quiroga, C. R. Faz, y R. J. L. Marquez. (2010). Estiercol de bovino lechero sobre el rendimiento y recuperacion aparente de nitrogeno en maiz forrajero. Terra Latinoamericana, 28 (4), 361-369.

Gallardo Lancho, J. F. (2001). Mineralización y humificación de la materia orgánica del suelo: 
Influencia del estiércol composteado y micorriza arbuscular sobre la composición química del suelo y el rendimiento productivo de maíz forrajero (Zea mays L.)

consecuencias sobre la contaminación. En Pérez, J. C. C., L. Alvarez, y N. W. Osorio (Eds.), X Congreso de la sociedad colombiana de la ciencia del suelo (pp. 141-153). Medellín, Colombia: Sociedad Colombiana de la Ciencia del Suelo.

Garcés, M. A. M., R. L. Berrio, A. S. Ruíz, J. G. Serna de León, y A. A. F. Builes. (2004). Ensilaje como fuente de alimentación para el ganado. Revista Lasallista de Investigación, $1(1), 66-71$.

García, G. A., M. P. Bernal, A. Roig. (2005). Organic matter fractions involved in degradation and humification processes during composting. Compost Science Utilization, 13, 127135.

García, E. (2004). Modificaciones al sistema de clasificación climática de Köppen (5a ed.). México: Instituto de geografía- Universidad Nacional Autónoma de México.

Gil, M. V., M. T. Carballo, y L. F. Calvo. (2008). Fertilization of maize with compost from cattle manure supplemented with additional mineral nutrients. Waste Management, 28 (8), $1432-1440$.

Gliessman, S. R., M. F. J. Rosado, Z. C. Guadarrama, J. Jedlicka, V. E. Mendez, R. Cohen, L Trujillo, C. Bacon, A. Cohn y R. Jaffe. (2007). Agroecología: promoviendo una transición hacia la sostenibilidad. Ecosistemas, 16 (1), 13-23.

Granados-Rivera, L. D., J. Quiroz-Valiente, J. A. Maldonado-Járquez, L. Granados-Zurita, P. Díaz-Rivera, y J. Oliva-Hernández, (2018). Caracterización y tipificación del sistema doble propósito en la ganadería bovina del Distrito de Desarrollo Rural 151, Tabasco, México. Acta Universita, 28 (6), 47-57.

López, M. J. D., E. A. Díaz, R. E. Martínez, y C. R. D. Valdez. (2001). Abonos orgánicos y su efecto en propiedades físicas y químicas del suelo y rendimiento en maíz. Terra Latinoamericana, 19 (4), 293-299.

López-Calderon, M. J., U. Figueroa-Viramontes, M. Fortis-Hernández, G. Núñez-Hernández, E. Ochoa-Martínez, y J. I. Sanchez-Duarte. (2015). Evaluación de dosis equivalentes de fertilizante y estiércol en la producción de maíz forrajero (Zea mays). Phyton, 84 (1), 813.

López-Martínez, J. D., C. Vázquez-Vázquez, E. Salazar-Sosa, R. Zúñiga-Tarango, y H. I. TrejoEscareño. (2010). Sistemas de labrabza y fertilización en la producción de maíz forrajero. Phyton-International Journal of Experimental Botany, 79 (871), 47-54. 
Martín, G. M., y R. Rivera. (2015). Influencia de la inoculación micorrízica en los abonos verdes. Efecto sobre el cultivo principal. Estudio de caso: el maíz. Cultivos Tropicales, 36(especial), 34-50.

Martínez, H. E., E. J. P. Fuentes, y H. E. Acevedo. (2008). Carbono orgánico y propiedades del suelo. Revista de la Ciencia del Suelo y Nutrición Vegetal, 8 (1), 68-96.

Monaco, S., D. J. Hatch, D. Sacco, C. Bertora, y C. Grignani. (2008). Changes in chemical and biochemical soil properties induced by 11-yr repeated additions of different organic materials in maize-based forage systems. Soil Biology and Biochemistry, 40, 608-615.

Montaño, A. N. M., G. V. Quiroz, y F. G. Cruz. (2001). Colonización micorrízica arbuscular y fertilización mineral de genotipos de maíz y trigo cultivados en un Andisol. Terra Latinoamericana, 19 (4), 337-344.

Osorio, N. W. (2012). pH del suelo y disponibilidad de nutrientes. Manejo Integral del Suelo y Nutrición Vegetal, 1 (4), 1-4.

Palma, L. D. J., D. J. Cisneros, C. E. Moreno, y R. J. A. Rincon. (2007). Suelos de Tabasco: Su Uso y Manejo Sustentable. (Colegio de Postgraduados, Ed.) (1ra ed.). Villahermosa, Tabasco: Colegio de Postgraduados, Instituto para el Desarrollo de Sistemas de Producción del Trópico Húmedo de Tabasco, Fundación Produce Tabasco A. C.

Pérez, L. Y. del C. (2012). Impacto de la biofertilización y aplicación de abonos orgánicos en la productividad de maíz (Zea mays L.) en Chiapas. Tesis de doctorado, El Colegio de la Frontera Sur, San Cristóbal de Las Casas, Chiapas, México. 125 p.

Phillips, J. M. y D. J. Hayman. 1970. Improved procedures for clearing and staining.

Pigurina, G. y G. E. Pérez. (1994). Momento de cosecha de maíz para ensilar. Montevideo, Uruguay: Instituto Nacional de Investigación Agropecuaria - URUGUAY.

Pimienta, B. E., H. J. Zañudo, y A. E. López. (2009). Efecto de las micorrizas arbúsculares en el crecimiento, fotosíntesis y anatomía foliar de plantas jóvenes de Agave tequiliana. Acta Botánica Mexicana, 89, 63-78.

Quiroga-Garza, H. M., J. A. Cueto-Wong, y U. Figueroa-Viramontes. (2011). Efecto del estiércol y fertilizante sobre la recuperación de $15 \mathrm{~N}$ y conductividad eléctrica. Terra Latinoamericana, 29 (2), 201-209.

Ramos, A. D. y A. E. Terry. (2014). Generalidades de los abonos orgánicos: importancia del bocashi como alternativa nutricional para suelos y plantas. Cultivos Tropicales, 35 (4), 
Influencia del estiércol composteado y micorriza arbuscular sobre la composición química del suelo y el rendimiento productivo de maíz forrajero (Zea mays L.)

$52-59$.

Salazar, S. E., E. H. I. Trejo, M. J. D. López, V. C. Vázquez, C. J. S. Serrato, I. Orona Castillo, y M. J. P. Flores. (2010). Efecto residual de estiercol bovino sobre el rendimiento de maíz forrajero y propiedades del suelo. Terra Latinoamericana, 28 (4), 381-390.

Salazar-Sosa, E., H. I. Trejo-Escareño, C. Vázquez-Vázquez, y J. D. López-Martínez. (2007). Producción de maíz bajo riego por cintilla, con aplicación de estiércol bovino. Phyton, 76, $169-185$.

Salazar-Sosa, E., H. I. Trejo-Escareño, C. Vázquez-Vázquez, J. D. López-Martínez, M. FortisHernández, R. Zuñiga-Tarango, y J. P. Amado-Álvarez. (2009). Distribución de nitrógeno disponible en suelo abonado con estiércol bovino en maíz forrajero. Terra Latinoamericana, 27 (4), 373-382.

Sánchez, S., M. Hernández, y F. Ruz. (2011). Alternativas de manejo de la fertilidad del suelo en ecosistemas agropecuarios. Pastos y Forrajes, 34 (4), 375-392.

SAS (Statistical Analysis Software). 2013. Institute Inc. User's guide. Statistics. Versión 8. Sixth edition. SAS Inc. Cary North Carolina, USA.

Secretaria de Medio Ambiente y Recursos Naturales (SEMARNAT) NOM-021-RECNAT-2000. (Norma Oficial Mexicana) (2002). Especificaciones de fertilidad, salinidad y clasificación de suelos. Estudio, muestreo y análisis. México. http://biblioteca.semarnat.gob.mx/janium/Documentos/Ciga/libros2009/DO2280n.pdf (22 de noviembre de 2018).

Setia, R., P. Marschner, J. Baldock, D. Chittleborough, P. Smith, y J. Smith. (2011). Salinity effects on carbon mineralization in soils of varying texture. Soil Biology and Biochemistry, 43 (9), 1908-1916.

Shanahan, J. F., N. R. Kitchen, W. R. Raun, y J. S. Schepers. (2008). Responsive in-season nitrogen management for cereals. Computers and Electronics in Agriculture, 61 (1), 5162.

SIAP (Servicio de Información Agroalimentaria y Pesquera). (2018). Estadística de producción agrícola. http://infosiap.siap.gob.mx/gobmx/datosAbiertos.php (27 de noviembre de 2018).

Smith S. E., y D. Read. (2008). Growth and carbon economy of arbuscular mycorrhizal symbionts. En: Press A, editor. Mycorrhizal symbiosis. Third Edit. London, UK: Elsevier 
B.V.p. 787.

Sparling, G. P., D. Wheeler, E. T. Vesely, y L. A. Schipper. (2006). What is soil organic matter worth? Journal of Environmental Quality, 35, 548-557.

Tinoco, A. C. A., M. F. A. Rodriguez, R. J. A. Sandoval, F. S. Barrón, C. A. Palafox, E. V. A. Esqueda, M. M. Sierra, y M. J. Romero. (2002). Manual de Produccion de Maiz para los Estados de Veracruz y Tabasco. $1^{a}$ edición. Veracruz, México: Instituto Nacional de Investigación Forestal, Agrícola y Pecuaria - Campo Experimental Papaloapan.

Trejo-Escareño, H. I., E. Salazar-Sosa, J. D. López-Martínez, y C. Vázquez-Vázquez. (2013). Impacto del estiércol bovino en el suelo y producción de forraje de maíz. Revista Mexicana de Ciencias Agrícolas, 4 (5), 727.

Trinidad, S. A. (2000). Utilización de estiércoles. Ficha Técnica. Montecillo, Edo de México.

Uribe, V. G., J. Petit, y E. R. Dzib. (2007). Respuesta del cultivo de maíz a la aplicación de biofertilizantes en el sistema roza, tumba y quema en suelo Alfisol (Chac-Lu 'Um, nomenclatura Maya), en Yucatán, México. Agricultura Andina, 13, 3-18.

West, R. C., N. P. Psuty, y B. G. Thom. (1985). Las tierras bajas de Tabasco (2da ed.). Villahermosa, Tabasco. Gobierno del estado de Tabasco.

Wiersma, D. W., P. R. Carter, K. A. Albrecht, y J. G. Coors. (1993). Kernel milkline stage and corn forage yield, quality, and dry matter content. Journal of Production Agriculture, 6 (1), 94-99.

Yan, N., P. Marschner, W. Cao, C. Zuo, y W. Qin. (2015). Influence of salinity and water content on soil microorganisms. International Soil and Water Conservation Research, 3, 316-323.

Zech, W., N. Senesi, G. Guggenberger, K. Kaiser, J. Lehmann, T. M. Miano, A. Miltner, y G. Schroth. (1997). Factors controlling humification and mineralization of soil organic matter in the tropics. Geoderma, 79 (1-4), 117-161. 